\title{
A Prediction Method for Acoustic Intensity Vector Field of Elastic Structure in Shallow Water Waveguide
}

\author{
Wenbo Wang $\mathbb{D},^{1}$ Desen Yang $\mathbb{D},^{1,2,3}$ and Jie Shi $\mathbb{D}^{1,2,3}$ \\ ${ }^{1}$ College of Underwater Acoustic Engineering, Harbin Engineering University, Harbin 150001, China \\ ${ }^{2}$ Key Laboratory of Marine Information Acquisition and Security, Harbin Engineering University, \\ Ministry of Industry and Information Technology, Harbin 150001, China \\ ${ }^{3}$ Acoustic Science and Technology Laboratory, Harbin Engineering University, Harbin 150001, China \\ Correspondence should be addressed to Jie Shi; shijie@hrbeu.edu.cn
}

Received 21 November 2019; Revised 9 February 2020; Accepted 12 February 2020; Published 19 October 2020

Academic Editor: Mahmoud Bayat

Copyright (C) 2020 Wenbo Wang et al. This is an open access article distributed under the Creative Commons Attribution License, which permits unrestricted use, distribution, and reproduction in any medium, provided the original work is properly cited.

\begin{abstract}
Compared with scalar sound field, vector sound field explained the spatial structure of sound field better since it not only presents the sound energy distribution but also describes the sound energy flow characteristics. Particularly, with more complicated interaction among different wavefronts, the vector sound field characteristics of an elastic structure in a shallow water waveguide are worthy of studying. However, there is no reliable prediction method for the vector sound field of an elastic structure with a high efficiency in a shallow water waveguide. To solve the problem, transfer functions in the waveguide have been modified with some approximations to apply for the vector sound field prediction of elastic structures in shallow water waveguides. The method is based on the combined wave superposition method (CWSM), which has been proved to be efficient for predicting scalar sound field. The rationality of the approximations is validated with simulations. Characteristics of the complex acoustic intensity, especially the vertical components are observed. The results show that, with constructive and destructive interferences in the depth direction, there could be quantities of crests and vortices in the spatial structure of time-dependent complex intensity, which manifest a unique dynamic characteristic of sound energy. With more complicated interactions among the wavefronts, a structure source could not be equivalent to a point source in most instances. The vector sound field characteristics of the two sources could be entirely different, even though the scalar sound field characteristics are similar. Meanwhile, source types, source parameters, ocean environment parameters, and geo parameters may have influence on the vector sound field characteristics, which could be explained with the normal mode theory.
\end{abstract}

\section{Introduction}

It has been a hot issue that how to precisely compute the radiated sound field of an underwater finite elastic structure. Particularly, in shallow seas such as the costal waters of China where the depth is less than 200 meters, the reflection effect from the boundaries could not be neglected [1]. Therefore, it is of great significance to study the radiated sound field in shallow water waveguides.

So far, researchers have presented numerous computing methods and analyses of radiated noise of a structure in a nonfree field. Wu defined a distance criterion for ray theory and the normal mode theory when computing the sound field of structures in an ideal waveguide by using the boundary element method (BEM) [2]. Bai et al. established a computing model of a two-dimensional structure in a simplified waveguide with the analytical method [3]. Li et al., Guo et al., and Miao et al. computed the radiated noise of cylindrical shells in nonfree fields based on an analytical method and the BEM [4-7]. Meanwhile, they analyzed the fluctuation characteristics of the sound field and the vibration of the structures in shallow water waveguides [8]. All the above studies indicate that reflection effects are not negligible in a nonfree field. However, some shortages such as large computational cost and singularity make the methods difficult to put into practice.

In 1989, Koopmann and Fahnline [9] presented the wave superposition method (WSM), which indicated that 
a structure could be equivalent to a series of virtual sources inside the structure. Later, lots of studies proved the efficiency of the WSM and found that the WSM avoids the nonuniqueness and singularity in the BEM with a higher computation speed [10-12]. Also, the factors affecting the accuracy of the WSM and the optimal strategies of equivalent source disposition were studied $[13,14]$. Soon, the WSM was introduced into a nonfree field. Some researchers investigated the sound field of a submerge structure with a finite depth by adjusting Green's function in the WSM $[15,16]$. Chen presented a field matching wave superposition method to minimize the quantity of equivalent sources. Meanwhile, he presented a quick computing method based on the WSM with some approximations [17]. In addition, to deal with the problem of multifield coupling in the shallow sea, Qian proposed a combined wave superposition method (CWSM), which combines the traditional wave superposition method with transfer functions in shallow water waveguides and the multiphysics field coupling numerical model. Then, he computed the sound field of structures in waveguides and found that, with the increase of the frequency, the difference between a point source and a structure is gradually obvious $[18,19]$.

It could be seen that most of the previous research studies were carried out on scalar sound field. In practice, the studies of vector sound field could provide us more profound understanding about the dynamic characteristics of the sound power, especially in shallow water waveguides. Therefore, the studies are significant and worthy of attention. For instance, some scholars investigated the rules of sound propagation in shallow water waveguides [20-24]. Meanwhile, some research studies on vector signal processing were also presented [25-27]. The estimation methods and analyses of sound energy provided more profound understand of dynamic characteristics of sound energy [28-31]. In addition, scholars studied the spatial structures of acoustic intensity which derives from interferences in waveguides [32, 33]. Though there are few studies about the vector sound field of structures in waveguides, the above works could still be beneficial references.

The contribution of this paper is that it proposed a feasible prediction method for the vector sound field of elastic structures in shallow water waveguides. Compared with traditional methods, the new method has an advantage in computational efficiency. Meanwhile, the new method solves the difficulties of acquiring the vibration information of an elastic structure in a complicated fluid environment. By analyzing the characteristics of the acoustic intensity vector field, the rule of energy flow could be obtained. The similarities and differences are analyzed by comparing the vector sound field of a point source and a structure source. In addition, this paper also analyzes the influences of ocean environmental parameters, source parameters, and geo parameters on the vector sound field of the structure source. The conclusions of the paper could provide priori information for sound field prediction, vector signal processing, and noise measurement.

\section{Theoretical Model}

The shallow sea environment is regarded as a Pekeris waveguide. The depth of the water layer is $H$ and the densities of water and the seabed are $\rho_{0}$ and. $\rho_{1}$. The compression wave speeds in the water and the seabed are $c_{0}$ and $c_{1}$. A simply supported water-loaded cylindrical shell with thickness $h$, radius $R$, length $L$, vacuo bulk mass density $\rho_{S}$, Young's modulus $E_{0}$, and the Poisson ratio $v$ is considered for the following analyses. The cylindrical shell is excited by a harmonic point force $F$, with the compute frequency $f_{0} . k_{0}$ is the wave number in the water and satisfies $k_{0}=2 \pi f_{0} / c_{0}$. The schematic diagram of the waveguide and the coordinate system is shown in Figure 1(a), and the sketch map of the cylindrical shell are shown in Figure 1(b).

2.1. Transfer Function in a Shallow Water Waveguide. In shallow water waveguides, the normal mode theory and the ray theory are used most commonly. In Chen's work, he indicated that, considering the calculation amount, the ray theory is more appropriate for computing the sound field near the source. On the contrary, the normal mode theory surpasses the ray theory for a longer distance [17].

In a Pekeris waveguide, the transfer function could be written with the two theories. For the simplicity of expression, only the transfer functions with a liquid seabed will be given in this character. The transfer functions for a waveguide with an elastic seabed will be given in the appendix.

2.1.1. Normal Mode Theory. The transfer function is Green's function in a Pekeris waveguide, which could be written as follows:

$$
\mathbf{g}_{N}(r, z)=-\frac{j}{4} \sum_{n} A_{n}^{2} \sin \left(k_{\mathrm{zn}} z\right) \sin \left(k_{\mathrm{zn}} z_{0}\right) H_{0}^{(2)}\left(\xi_{n} r\right), \quad 0<z \leq H,
$$

where $H_{0}^{(2)}(\cdot)$ is the second kind of zero-order Hankel function. $k_{\mathrm{zn}}$ and $\xi_{n} \xi_{n}$ are the $n$th eigenvalues in the vertical and horizontal directions which satisfy $k_{0}^{2}=\xi_{n}^{2}+k_{\mathrm{zn}}^{2} . A_{n}^{2}$ is the $n$th modal amplitude which could be written as follows:

$$
A_{n}^{2}=\frac{2 k_{\mathrm{zn}}}{k_{\mathrm{zn}} H-\sin \left(k_{\mathrm{zn}} H\right) \cos \left(k_{\mathrm{zn}} H\right)-\left(\rho_{0} / \rho_{1}\right)^{2} \sin ^{2}\left(k_{\mathrm{zn}} H\right) \tan \left(k_{\mathrm{zn}} H\right) .}
$$

\subsubsection{Ray Theory. The transfer function is}

$$
\mathbf{g}_{R}(r, z)=\frac{e^{-j k_{0} R_{0}}}{4 \pi R_{0}}+\sum_{i=1}^{\bar{n}}\left(F_{U \bar{n}}^{m} F_{L \bar{n}}^{i-m} \frac{e^{-j k_{0} R_{U \bar{n}}}}{4 \pi R_{U \bar{n}}}+F_{L \bar{n}}^{m} F_{U \bar{n}}^{i-m} \frac{e^{-j k_{0} R_{L \bar{n}}}}{4 \pi R_{L \bar{n}}}\right),
$$

where $m=(\bar{n} / 2)+\left[1+(-1)^{\bar{n}+1}\right] / 4, \bar{n}$ is the number of reflection times, $j=\sqrt{-1} . R_{0}$ is the distance between the source and the field point. $R_{U \bar{n}}$ is the distance between the $n$-order image source beyond the top of the waveguide and the field 


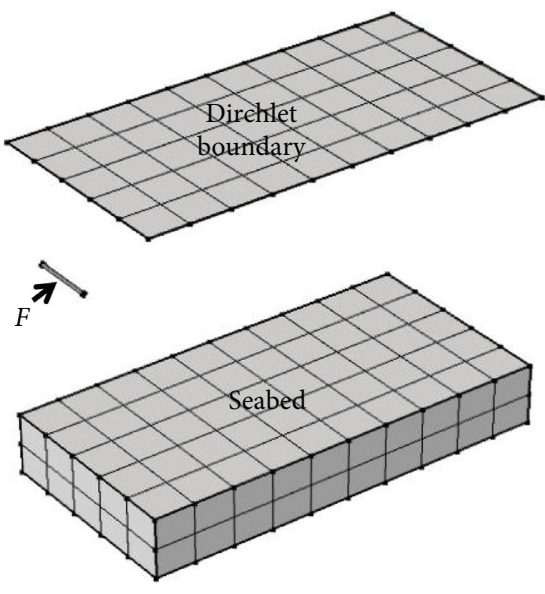

(a)

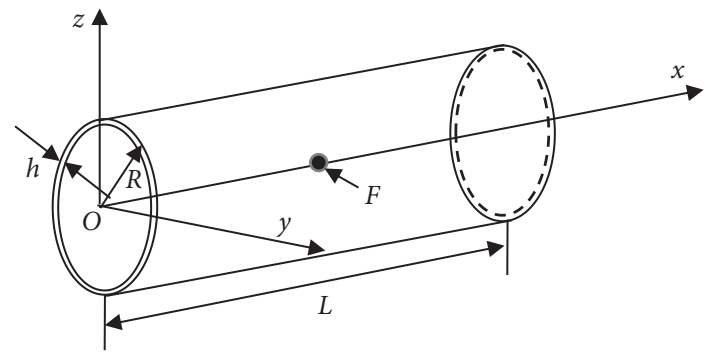

(b)

Figure 1: (a) The acoustic radiation model of structure in a Pekeris waveguide. (b) The sketch map of the cylindrical shell with finite length.

point. $R_{L \bar{n}}$ is the distance between the $n$-order image source beneath the waveguide bottom and the field point. The $z$-coordinate of the image sources beyond the surface $z_{U \bar{n}}$ and the ones beneath the bottom $z_{L \bar{n}}$ are written as follows:

$$
\begin{aligned}
& z_{U n}=\left(2 z_{0}+h_{L}-h_{U}\right) \cdot \frac{1+(-1)^{\bar{n}+1}}{2}-\bar{n} H-z_{0}+h_{U}, \\
& z_{L n}=\left(2 z_{0}+h_{L}-h_{U}\right) \cdot \frac{1+(-1)^{\bar{n}+1}}{2}+\bar{n} H-z_{0}+h_{U},
\end{aligned}
$$

where $z_{0}$ is the $z$-coordinate of the source. $h_{U}$ is the submerge depth of the source and $h_{L}=H-h_{U}$.

In a Pekeris waveguide, the top boundary is soft and the reflection coefficient is defined as $F_{U \bar{n}}=-1$. The reflection coefficient of the lower boundary $F_{L \bar{n}}$ varies with $\bar{n}$ and $F_{L \bar{n}}=F_{0}+B . F_{0}$ and $B$ are two functions of $\theta_{\bar{n}}$, where $\theta_{\bar{n}}=\arctan \left[\left(z_{L \bar{n}}-z_{0}\right) / r\right]$, which is defined as the grazing angle. The expressions of $F_{0}$ and $B$ are shown as below:

$$
\begin{aligned}
F_{0} & =\frac{M \sin \theta_{\bar{n}}-\sqrt{\eta^{2}-\cos ^{2} \theta_{\bar{n}}}}{M \sin \theta_{\bar{n}}+\sqrt{\eta^{2}-\cos ^{2} \theta_{\bar{n}}}}, \\
B & =\frac{\left(\left(\partial^{2} F_{0} / \partial \theta_{\bar{n}}\right)-\left(\partial F_{0} / \partial \theta_{\bar{n}}\right) \tan \theta_{\bar{n}}\right)}{2 j k_{0} R_{L \bar{n}}},
\end{aligned}
$$

where $M=\rho_{0} / \rho_{1}$ and $\eta=c_{1} / c_{0}$.

When considering an elastic seabed, there are shear waves in the seabed, and the expression of $\mathbf{g}_{N}(r, z)$ and reflection coefficient of the seabed $V$ are available in the appendix.

In a shallow water waveguide, the frequency threshold for the ray theory when the acoustic beam has formed is $f_{c}=8 c_{0} / H$. Actually, for computing the sound field of an elastic structure in the waveguide where the acoustic beam has not formed, the threshold could be much lower [17].

2.2. Wave Superposition Method in a Shallow Water Waveguide. According to section 2.1, the schematic diagram of the CWSM and the computing model could be shown as Figure 2:
The theoretical basis of the WSM is that the combined fields of a series of virtual sources inside the structure could be made to reproduce the structural vibration. The intensity matrix of the virtual sources is $\mathbf{q}=[\mathbf{D}]^{-1}[\mathbf{U}] \cdot[\mathbf{D}]$ is defined as the dipole matrix which describes the relationship between the equivalent source intensities and the vibration information on the structure surface and $[\mathbf{D}]=\nabla_{n} \mathbf{g}\left(\left|\overrightarrow{\mathbf{r}_{\mathbf{s}}}-\overrightarrow{\mathbf{r}_{0}}\right|\right)$. [U] is the normal velocity vector of the structure surface nodes. $[\mathbf{D}]^{-1}$ is the generalized inverse matrix of $[\mathbf{D}]$. Meanwhile, some regularization methods are adopted to avoid ill-conditioned matrix when the dimension of [D] is large [13]. The process of regularization could bring in some extra computational cost which is a shortcoming for the WSM.

Afterwards, the sound pressure vector $[\mathbf{P}]$ of field points is

$$
[\mathbf{P}]=[\mathbf{T}] \mathbf{q}=[\mathbf{T}][\mathbf{D}]^{-1}[\mathbf{U}],
$$

where $[\mathbf{T}]$ is the transfer matrix which could be written as follows:

$$
[\mathbf{T}]=j \rho_{0} \omega_{0} \mathbf{g}\left(\left|\vec{r}-\overrightarrow{\mathbf{r}_{0}}\right|\right),
$$

where $\overrightarrow{\mathbf{r}_{0}}, \vec{r}$, and $\overrightarrow{\mathbf{r}_{\mathbf{s}}}$ represent the locations of equivalent sources, observation points, and nodes on the structure surface.

2.3. Acquisition of Structural Vibration. The reflected sounds from the waveguide boundaries affect the sound field and vibration of a structure. However, acoustic-solid couplings and fluid-solid couplings make it arduous to acquire the vibration information on the structure surface with analytical methods. Likewise, it is costly to obtain the vibration information with experimental methods. According to the work of Qian [18], the finite element method (FEM) could be applied for establishing the numerical model for computing the structural vibration in a shallow water waveguide with a tiny computational cost at low frequencies. The numerical model could be shown as Figure 3(a). 


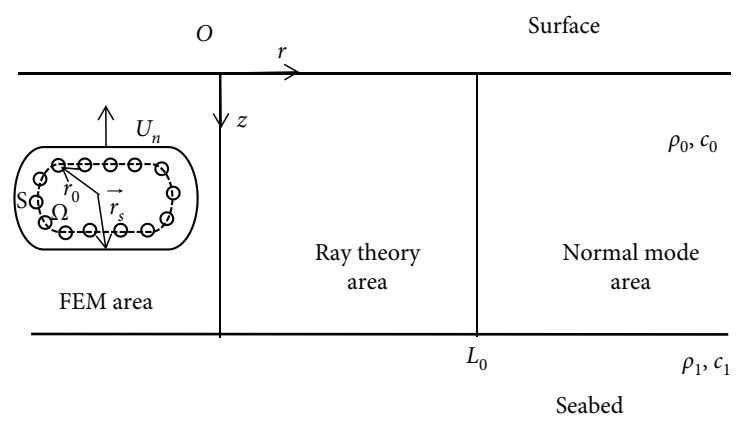

(a)

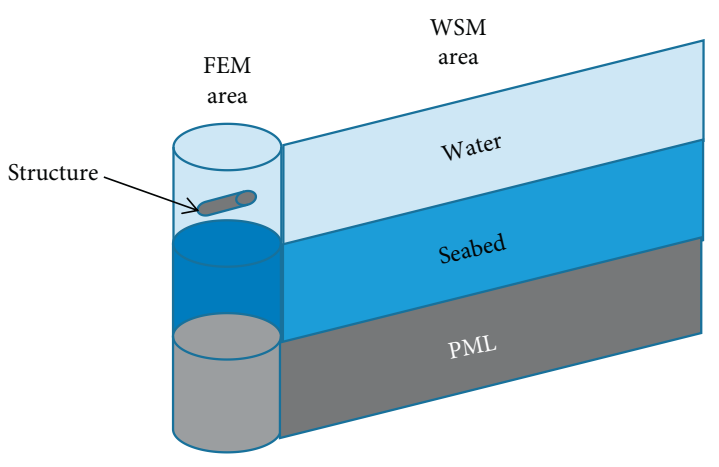

(b)

Figure 2: (a) Principle of combined wave superposition method in a shallow water waveguide. (b) Model of a cylindrical shell sound radiation based on CWSM.

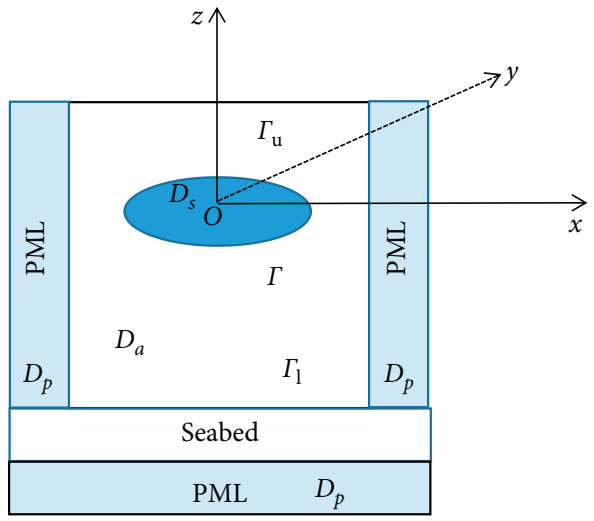

(a)

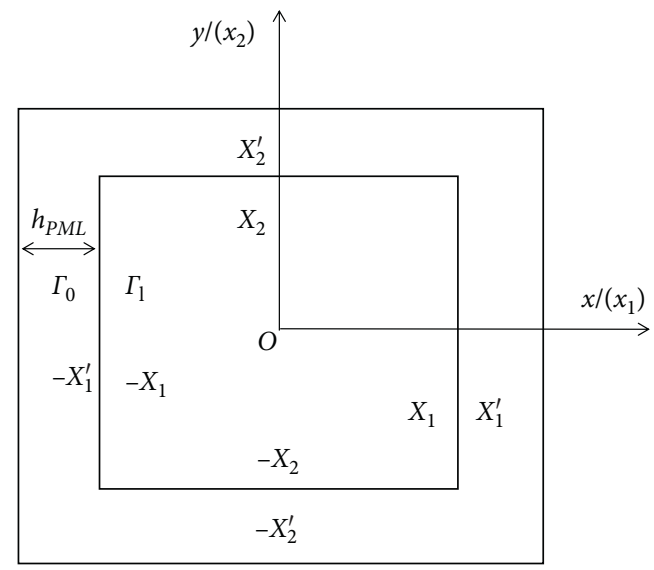

(c)

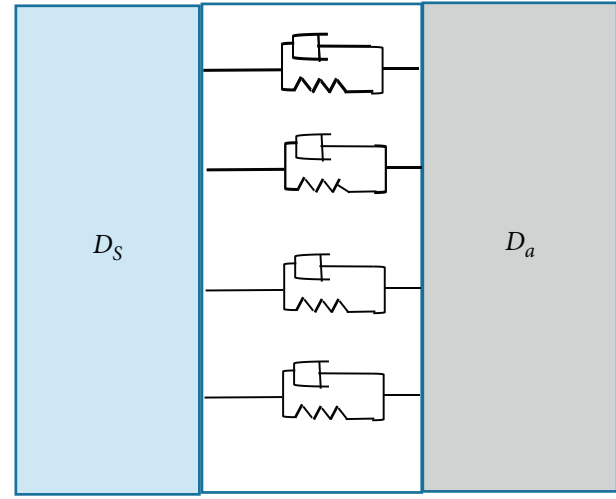

(b)

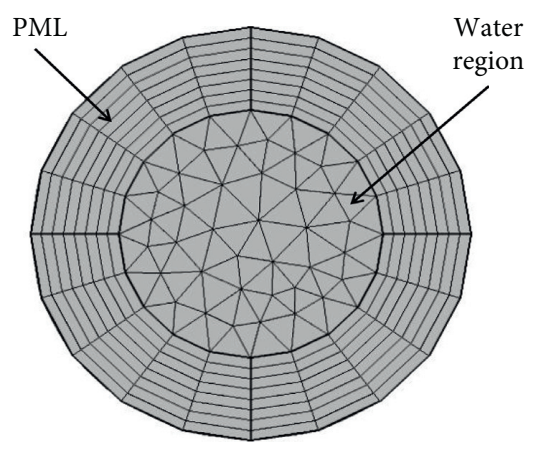

(d)

Figure 3: (a) The numerical model of multiphysical field with an elastic structure in a shallow water waveguide. (b) Fluid-solid coupling model. (c) The model of acoustic perfectly matched layer. (d) FEM area from the XY aspect.

In the model, $D_{s}$ is the fluid domain inside the structure, $D_{a}$ is the fluid domain in the waveguide, and $D_{P}$ is the domain of perfect matched layer(PML), which satisfies that there is no reflected sound from the infinity. $\Gamma_{I}$ and $\Gamma_{O}$ are the inner and outer boundaries of the PML, respectively, as shown in Figure 3(c). The thickness of the PML is $h_{\mathrm{PML}} \cdot \Gamma_{u}$ is the interface of the sound field and the top boundary of the waveguide, while $\Gamma_{l}$ is the interface of the sound field and the lower boundary of the waveguide. $\Gamma$ is the interface of $D_{a}$ and the structure.

According to the theory of the FEM, for an elastic structure, the vibration equation could be written as follows: 


$$
\left(\mathbf{K}_{\mathbf{S}}+j \omega \mathbf{C}_{\mathbf{S}}-\omega_{0}^{2} \mathbf{M}_{\mathbf{S}}\right)\left\{\mathbf{u}_{\mathbf{i}}\right\}=\left\{\mathbf{F}_{\mathbf{S i}}\right\},
$$

where $\mathbf{M}_{\mathbf{S}}, \mathbf{K}_{\mathbf{S}}$, and $\mathbf{C}_{\mathbf{S}}$ are the mass matrix, stiffness matrix, and damping matrix of the structural mesh. $\left\{\mathbf{F}_{\mathrm{Si}}\right\}$ is the exciting load on the structure, and $\left\{\mathbf{u}_{\mathbf{i}}\right\}$ is the normal velocity vector on $\Gamma$.

In a similar way, the acoustical FEM equation in the fluid domain is

$$
\left(\mathbf{K}_{\mathbf{a}}+j \omega_{0} \mathbf{C}_{\mathbf{a}}-\omega_{0}^{2} \mathbf{M}_{\mathbf{a}}\right)\left\{\mathbf{p}_{i}\right\}=\left\{\mathbf{F}_{\mathbf{i}}\right\},
$$

where $\mathbf{M}_{\mathbf{a}}, \mathbf{K}_{\mathbf{a}}$, and $\mathbf{C}_{\mathbf{a}}$ are the mass matrix, stiffness matrix, and damping matrix of the fluid mesh. $\left\{\mathbf{p}_{i}\right\}$ represents the pressure vector on $\Gamma$, and $\left\{\mathbf{F}_{\mathbf{i}}\right\}$ is the acoustic excitation.

As shown in Figure 3(b), the normal velocity of the structure $v_{\text {sn }}$ should be equal to the normal velocity $v_{\text {an }}$ in $D_{a}$ :

$$
v_{\mathrm{an}}=-\frac{1}{j \omega \rho_{0}} \frac{\partial p_{i}}{\partial n}=v_{\mathrm{sn}}
$$

With equations (8) to (10), the fluid-solid coupling equation on $\Gamma$ could be written as follows:

$$
\left(\begin{array}{cc}
\mathbf{K}_{\mathbf{S}} & \mathbf{K}_{\mathbf{c}} \\
0 & \mathbf{K}_{\mathbf{a}}
\end{array}\right)+j \omega\left(\begin{array}{cc}
\mathbf{C}_{\mathbf{S}} & 0 \\
0 & \mathbf{C}_{\mathbf{a}}
\end{array}\right)-\omega_{0}^{2}\left(\begin{array}{cc}
\mathbf{M}_{\mathbf{S}} & 0 \\
-\rho_{0} \mathbf{K}_{\mathbf{c}}^{\mathrm{T}} & \mathbf{M}_{\mathbf{a}}
\end{array}\right)\left(\begin{array}{c}
u_{i} \\
p_{i}
\end{array}\right)=\left(\begin{array}{c}
F_{\text {st }} \\
F_{\text {at }}
\end{array}\right) .
$$

In equation (11), $F_{\text {st }}$ and $F_{\text {at }}$ are the coupled-field load for the structure and the fluid domain. $\mathbf{K}_{\mathbf{c}}$ and $\mathbf{M}_{\mathbf{c}}$ are coupling stiffness matrix and coupling mass matrix and $\mathbf{M}_{\mathbf{c}}^{\mathbf{T}}=-\rho_{0} \mathbf{K}_{\mathbf{c}}^{\mathbf{T}}$.

The PML equation in the frequency domain could be written as follows:

$$
\left\{\begin{array}{l}
p_{i}=-\frac{\rho_{0} c_{0}^{2}}{\sigma_{i}-\mathrm{j} \omega} \frac{\partial v_{i}}{\partial x_{i}}, \quad i=1 \text { or } 2, \text { in } D_{p}, \\
v_{i}=-\frac{1}{\rho_{0}\left(\sigma_{i}-\mathrm{j} \omega\right)} \frac{\partial p_{i}}{\partial x_{i}}, \quad i=1 \quad \text { or } 2, \text { in } D_{p} \\
p=p_{1}+p_{2},
\end{array}\right.
$$

where $\sigma_{i}$ is the absorptivity and $p_{i}$ and $v_{i}$ are the sound pressure and particle velocity in the PML region. With equation (12), the Helmholtz equation could be modified as the expression form below:

$$
k_{0}^{2} p+\sum_{i=1}^{2} \frac{\mathrm{j} \omega_{0}}{\sigma_{i}-\mathrm{j} \omega_{0}} \frac{\partial}{\partial x_{i}}\left(\frac{\mathrm{j} \omega_{0}}{\sigma_{i}-\mathrm{j} \omega_{0}} \frac{\partial p_{i}}{\partial x_{i}}\right)=0 .
$$

The spatial absorptivity is introduced into the model:

$$
\gamma_{i}\left(x_{i}\right)=\left\{\begin{array}{ll}
1, & \left|x_{i}\right|<X_{i}, \\
1+\frac{j}{\omega} \sigma_{i}\left(\left|x_{i}\right|\right), & X_{i} \leq\left|x_{i}\right|<X_{i}^{\prime}
\end{array} .\right.
$$

Thus, equation (13) could be rewritten as below:

$$
\gamma_{1} \gamma_{2} k_{0}^{2} p-\left[\frac{\partial}{\partial x_{1}}\left(\frac{\gamma_{2}}{\gamma_{1}} \frac{\partial p}{\partial x_{1}}\right)+\frac{\partial}{\partial x_{2}}\left(\frac{\gamma_{1}}{\gamma_{2}} \frac{\partial p}{\partial x_{2}}\right)\right]=0
$$

Generally, the thickness of the PML mesh should not be less than 1/15 of the wavelength, as shown in Figure 3(d). In addition, it is not formidable to simulate different kinds of boundaries such as rigid seabed, liquid seabed, and elastic seabed with flexible parameter settings.

2.4. Approximations in the Solution of the Source Intensity. In a shallow water waveguide, the normal velocity on the structure surface $\mathbf{U}$ consists of three parts considering reflected sounds from the waveguide boundaries: $\mathbf{U}_{\mathbf{n}}$ caused by the exciting force, $\mathbf{U}_{\mathbf{S}}$ caused by the scattered sounds, and $\mathbf{U}_{\mathbf{R}}$ caused by the reflection sounds. According to the work of Chen, $\mathbf{U}_{\mathbf{R}}$ does not contribute to the radiated sound field [17]. Therefore, $\mathbf{U}_{\mathbf{R}}$ should be separated out from the total velocity $\mathbf{U}$. The expression of sound pressure in the sound field could be written as follows:

$$
\mathbf{P}=j \rho_{0} \omega_{0} \mathbf{g}\left(\nabla_{n} \mathbf{G}\right)^{-1}\left(\mathbf{U}_{\mathbf{n}}+\mathbf{U}_{\mathbf{S}}\right)
$$

To some extent, $\mathbf{U}_{\mathbf{n}}+\mathbf{U}_{\mathbf{S}}$ derives from the equivalent sources inside the structure. Meanwhile, $\mathbf{U}_{\mathbf{R}}$ derives from the mirror images of the sources. Assume, $\mathbf{U}_{\mathbf{R U n}}, \mathbf{q}_{\mathbf{U n}}$, and $\mathbf{G}_{\mathbf{U n}}$ are the velocity matrix, density matrix, and Green function matrix which stem from the $n$-order image sources beyond the top of the waveguide, while $\mathbf{U}_{\mathbf{R L n}}, \mathbf{q}_{\mathbf{L n}}$, and $\mathbf{G}_{\mathbf{L n}}$ for the $\mathrm{n}$-order image sources beneath the bottom of the waveguide. The normal velocity of the structure surface could be separated as follows:

$$
\left\{\begin{array}{l}
\mathbf{U}_{\mathbf{n}}+\mathbf{U}_{\mathbf{S}}=\left(\nabla_{n} \mathbf{G}\right) \mathbf{q}, \\
\mathbf{U}_{\mathbf{R U n}}=\left(\nabla_{n} \mathbf{G}_{\mathbf{U n}}\right) \mathbf{q}_{\mathbf{U n}}, \\
\mathbf{U}_{\mathbf{R L n}}=\left(\nabla_{n} \mathbf{G}_{\mathbf{L n}}\right) \mathbf{q}_{\mathbf{L n}} .
\end{array}\right.
$$

According to the relationship between the equivalent sources and their images, $\mathbf{U}_{\mathbf{R U n}}$ and $\mathbf{U}_{\mathbf{R L n}}$ could be written as follows:

$$
\begin{gathered}
\mathbf{U}_{\mathbf{R U n}}=F_{U n}\left(\nabla_{n} \mathbf{G}_{\mathbf{U n}}\right)\left(\nabla_{n} \mathbf{G}\right)^{-1}\left(\mathbf{U}_{\mathbf{n}}+\mathbf{U}_{\mathbf{S}}\right), \\
\mathbf{U}_{\mathbf{R} \mathbf{n}}=F_{L n}\left(\nabla_{n} \mathbf{G}_{\mathbf{L n}}\right)\left(\nabla_{n} \mathbf{G}\right)^{-1}\left(\mathbf{U}_{\mathbf{n}}+\mathbf{U}_{\mathbf{S}}\right) .
\end{gathered}
$$

In the above formula, $\mathbf{G}$ is the Green function matrix in the free field which is much easier to solute partial derivatives than that for the Green function in a nonfree field. With equations (16) to (18), $\mathbf{U}_{\mathbf{n}}+\mathbf{U}_{\mathbf{S}}$, which contributes to the radiated sound, could be extracted from $\mathbf{U}$ :

$$
\mathbf{U}_{\mathbf{n}}+\mathbf{U}_{\mathbf{S}}=\left\{\mathbf{E}+\left[\sum_{i=1}^{M^{\prime}} F_{U}\left(\nabla_{n} \mathbf{G}_{\mathbf{U i}}\right)+\sum_{k=1}^{N^{\prime}} F_{L k}\left(\nabla_{n} \mathbf{G}_{\mathbf{L k}}\right)\right]\left(\nabla_{n} \mathbf{G}\right)^{-1}\right\}^{-1} \mathbf{U} .
$$

$M^{\prime}$ is the order number of the virtual sources beyond the top of the waveguide and $N^{\prime}$ for the virtual sources beneath the waveguide bottom to make computing result converge. 
In equation (19), the orders of the image sources should be truncated to accomplish the computation. Actually, with the increase of the order, the influence of the image sources on the normal velocity decrease rapidly. Therefore, only the first few orders of the image sources are necessary to be taken into consideration. Furthermore, if the minimum distance $D_{m}$ between the boundaries and the structure exceeds the maximum linear dimension $L_{M}$ of the structure, equation (19) could be rewritten as follows:

$$
\mathbf{P}=j \rho_{0} \omega_{0} \mathbf{g}\left(\nabla_{n} \mathbf{G}\right)^{-1} \mathbf{U}
$$

In the above case, the velocity variation caused by virtual sources is an order of magnitude lower than $\mathbf{U}_{\mathbf{n}}+\mathbf{U}_{\mathbf{S}}$, and $\mathbf{U} \approx \mathbf{U}_{\mathbf{n}}+\mathbf{U}_{\mathbf{S}}$ is satisfied. In fact, when $D_{m}$ and $L_{M}$ are comparable and the frequency is not very high, the effect of scattered sound could also be neglected. The above deductions will be validated with some simulations in the following text.

2.5. Acoustic Intensity. According to the Euler equation, the horizontal and vertical particle velocities could be computed with the gradients of sound pressure:

$$
\left\{\begin{array}{l}
v_{r}=\frac{1}{j \rho_{0} \omega_{0}} \frac{\partial p(r, z)}{\partial r} \\
v_{z}=\frac{1}{j \rho_{0} \omega_{0}} \frac{\partial p(r, z)}{\partial z}
\end{array}\right.
$$

Combining equation (21) with equation (7), the particle velocity vectors are as below:

$$
\left\{\begin{array}{l}
v_{r}(r, z)=\frac{\partial}{\partial r}[\mathbf{g}(r, z) \mathbf{q}], \\
v_{z}(r, z)=\frac{\partial}{\partial z}[\mathbf{g}(r, z) \mathbf{q}] .
\end{array}\right.
$$

In actual engineering, people usually use differential solutions instead of derivative solutions that equation (21) could be rewritten as follows:

$$
\left\{\begin{array}{l}
v_{r}\left(r_{0}, z_{0}\right) \approx \frac{p\left(r_{0}+\Delta r, z_{0}\right)-p\left(r_{0}, z_{0}\right)}{j \rho_{0} \omega_{0} \Delta r}, \\
v_{z}\left(r_{0}, z_{0}\right) \approx \frac{p\left(r_{0}, z_{0}+\Delta z\right)-p\left(r_{0}, z_{0}\right)}{j \rho_{0} \omega_{0} \Delta z},
\end{array}\right.
$$

where $\Delta r$ and $\Delta z$ are defined as distances along the horizontal or vertical direction which are much less than the wavelength. It is not difficult to find:

$$
\left\{\begin{aligned}
v_{r}\left(r_{0}, z_{0}\right) \approx \frac{\partial g(r, z)}{\partial r} \mid & \begin{array}{c}
r \\
\end{array}=r_{0} q \\
z & =z_{0} \\
v_{z}\left(r_{0}, z_{0}\right) \approx \frac{\partial g(r, z)}{\partial z} \mid & \\
r & =r_{0} q . \\
z & =z_{0}
\end{aligned}\right.
$$

Obviously, equation (24) establishes the relationship between the equivalent source intensity and particle velocities.

The time-dependent complex acoustic intensities could be written as $I_{C}(t)=(1 / 2) p(t) v^{*}(t)$, where the asterisk denotes the complex conjuration. And the time factor is $\exp \left(-j \omega_{0} t\right)$. The active intensity $I(t)$ is represented by the real part of the complex intensity and the reactive intensity $Q(t)$ is represented by the imaginary part of the complex intensity. The above two components describe the dynamic characteristics of the acoustic energy flux. For a free field, when $k_{0} r \gg 1, Q(t)$ tends to be zero. However, in a nonfree field, the multipath interference could be sources of the reactive intensity, and $Q(t)$ exists even at a long distance.

Jacobsen first put forward a concept of "normalized forms of the complex intensity," which were referred to as the pressure indices which could be applied for acquiring the nature of the intensity vector field [34]. The active pressure intensity index and the reactive pressure index are defined as

$$
\left\{\begin{array}{l}
I^{*}=\frac{I}{\left|p^{2}\right| / \rho_{0} c_{0}}, \\
Q^{*}=\frac{Q}{p^{2} / \rho_{0} c_{0}} .
\end{array}\right.
$$

The asterisk here does not represent a complex conjugation. Instead, it indicates that these are normalized intensity quantities. In a shallow water waveguide, the multipath interferences take place in the vertical direction. Hence, $I$ and $Q$ are readily manifested by their vertical components, $I_{z}^{*}$ and $Q_{z}^{*}$.

The spatial structure of acoustic intensity in a waveguide could be divided into two categories: crests and vortices, which are related to constructive interference and destructive interference, respectively.

In the regions of crests, there are maxima in the $\left|p^{2}\right|$ field and $\left|I_{z}^{*}\right|<1$ and $\left|Q_{z}^{*}\right|<1$. On the contrary, in the regions of vortices, minima exist in the $\left|p^{2}\right|$ field and $\left|I_{z}^{*}\right|>1,\left|Q_{z}^{*}\right|>1$. Therefore, active intensity has a left- or right-handed curl at the regions of vortices. Particularly, at the center of a vortex, there is a dislocation where both $\left|p^{2}\right|$ and $I_{C}$ are zero. At the same time, the reactive intensity converges toward the dislocation. Vortices in the acoustic intensity vector field have outstanding meanings in a shallow water waveguide. 
The unique characteristics at the vortices could have extensive usages in noise measurement, sound prediction, source localization, acoustical holography, etc.

\section{Numerical Analysis}

By establishing an acoustic intensity vector sound field computing model of an elastic cylinder in a Pekeris waveguide as shown in Figure 2, the parameter values for the numerical simulations are defined as follows: $L=10 \mathrm{~m}$, $R=1 \mathrm{~m}, h=0.01 \mathrm{~m}, E_{0}=2.0 \times 10^{11} \mathrm{~Pa}, \rho_{s}=7850 \mathrm{~kg} / \mathrm{m}^{3}$, $v=0.3, \rho_{0}=1000 \mathrm{~kg} / \mathrm{m}^{3}, c_{0}=1500 \mathrm{~m} / \mathrm{s}$, and $F=1 N$. In addition, the position of excitation is $\left(0,-R, h_{U}\right)$. The geometric center of the shell is $\left(0,0, h_{U}\right)$. The number of equivalent sources inside the cylindrical shell is 900 , and the retraction ratio is 0.6 with verification of simulations.

\subsection{The Equivalent Source Intensities with Reflections of} Boundaries. As discussed in Section 2.4, intensities of the equivalent sources inside the structure could be acquired with the ray theory. Meanwhile, some approximations could be employed to reduce computational cost.

With the increase of $\bar{n}$, the grazing angle $\theta_{\bar{n}}$ tends to be larger. In a Pekeris waveguide with an elastic seabed, the relationship between $\theta$ and the reflection coefficient $V$ could be shown in Figure 4. The influences of seismic wave speeds and seabed density are analyzed. It could be found that $|V|$ tends to be a constant as grazing angle $\theta$ increases. With the increase of $c_{p}$ and $c_{s}$, the seabed tends to be more 'rigid' and $|V|$ is larger at large grazing angles.

In the following simulations, different orders of image equivalent sources are taken into consideration for computing the equivalent source intensities.

For instance, when $i$ orders of image equivalent sources beyond the top boundary and $j$ orders of image equivalent sources beneath the bottom boundary are taken into account, the case is recorded as a $U_{i} D_{j}$ case. When $i$ and $j$ are large enough, the result converges to a constant. The corresponding serial number for different cases is shown in Table 1:

To measure the accuracy of the equivalent source intensities, two parameters are defined as average error ratios according to the amplitude error and phase error between the calculated values and the convergence values:

$$
\begin{aligned}
& \text { error1 }=\left[\frac{1}{\operatorname{Num}} \sum_{i=1}^{\text {Num }}\left(A_{i}^{q}-\overline{A_{i}^{q}}\right)\right] * 100 \%, \\
& \text { error2 }=\left[\frac{1}{\operatorname{Num}} \sum_{i=1}^{\text {Num }}\left(P_{i}^{q}-\overline{P_{i}^{q}}\right)\right] * 100 \%,
\end{aligned}
$$

where Num is the quantity of equivalent sources and $A_{i}^{q}$ and $P_{i}^{q}$ are the amplitude and phase of the equivalent source in every case. $\overline{A_{i}^{q}}$ and $\overline{P_{i}^{q}}$ are the convergence values when the orders are large enough.

When $f_{0}=120 \mathrm{~Hz}$, the accuracy of equivalent source intensities in different cases are analyzed. According to equations (19) and (20), with different submerge depths, the influences of the image sources are not the same. When $h_{U}=30 \mathrm{~m}$, the average error ratios could be neglected for almost all the cases. However, when $h_{U}=1.2 \mathrm{~m}$ or $h_{U}=58.8 \mathrm{~m}$, the source is quite close to the top boundary or the bottom boundary that the influences of reflected sounds are nonnegligible. Nevertheless, only first few cases are under the influence. As shown in Figure 5, when $N_{S}>5$, the average error ratios tend to be zero. Therefore, though a structure is quite close to the boundaries, the equivalent source intensities could also be computed precisely considering first few orders of image sources. Thus, the computational cost could be further reduced.

From another aspect, for a structure with regular shape such as a cylindrical shell, the reflection effects on the structural vibration could be shown in modal analyses. The reflected sounds bring in the variations of the structural vibration. Many factors such as the reflection coefficient of the seabed and the submerge depth of the structure may influence the details of the structural vibration.

Assume that the submerge depth is fixed to $2 \mathrm{~m}$, the vibration modes in different fluid environments could be shown in Table.2. It is important to point out that, in the following simulations, to reduce the computational cost, the depth of the water layer is fixed to $30 \mathrm{~m}$, which is not big enough and $D_{m}>L_{M}$ is not satisfied. Therefore, still finite orders of image sources should be considered to acquire precise equivalent source intensities and structural vibration information.

It could be seen in Table 2 that, in different fluid environments, the modal frequencies and modal shapes for the certain vibration modes are slightly different. However, the discrepancies may cause different vibration responses in different fluid environments with the same excitation, which means that the equivalent source intensities could be entirely different. Therefore, when the structure is close to a boundary of the waveguide, the influences of reflected sounds on the structural vibration should be considered. In addition, it could be found that, in the half-space, the modal frequencies are always slightly higher than those in the free field. The phenomenon could be explained that, with a Dirichlet boundary, the reflected sounds from the soft boundary reduce the added mass of water to the structure, which increases the modal frequencies. On the contrary, when the structure is in a waveguide, the reflected sounds from the seabed raise the added mass of water to the structure and make the modal frequencies lower.

Figure 6 shows the modal frequencies for the four chosen modes with the variation of $h_{U}$. With the increase of $h_{U}$, the modal frequency of the certain mode fluctuates around the modal frequency for free field. Thus, the vibration response also changes with the variation of $h_{U}$. In an ideal waveguide with a soft top boundary and a rigid seabed, the fluctuation is more volatile than that in a Pekeris waveguide. Meanwhile, when the structure is close to the seabed, the modal frequencies begin to decrease and the trend for the ideal waveguide seem to be more obvious. It is mainly because that the reflection coefficient for a rigid boundary is larger than a liquid seabed. Thus, the reflection effect in the ideal waveguide is much stronger than that in a Pekeris waveguide. 
Surface

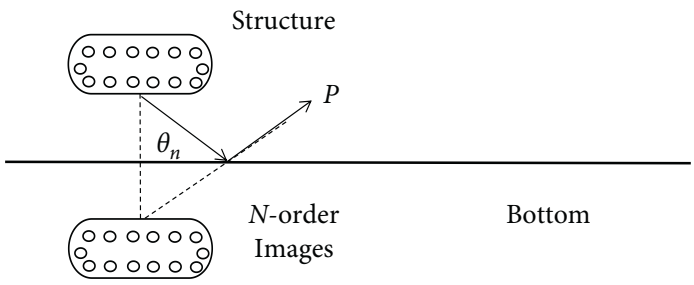

(a)

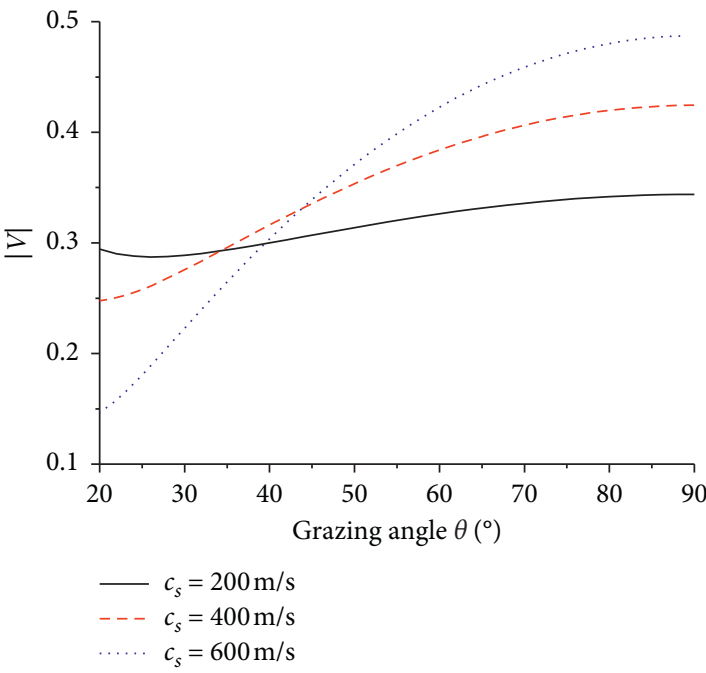

(c)

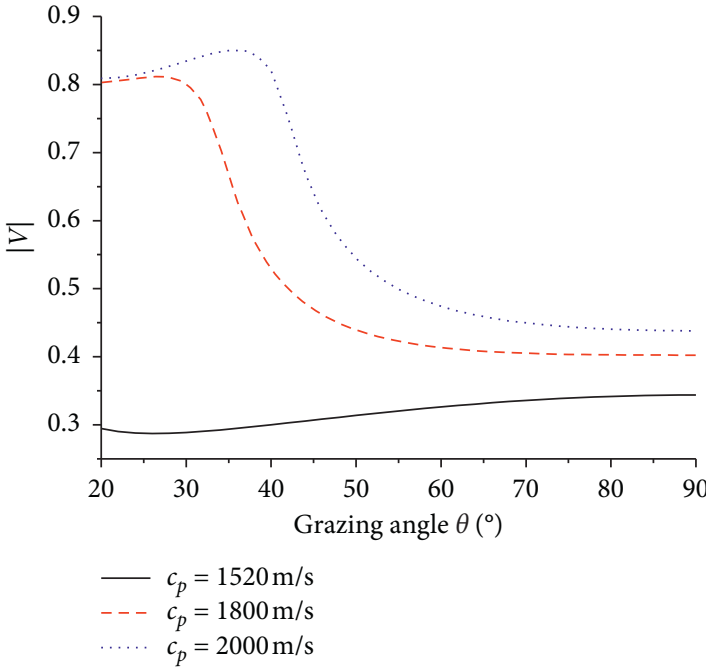

(b)

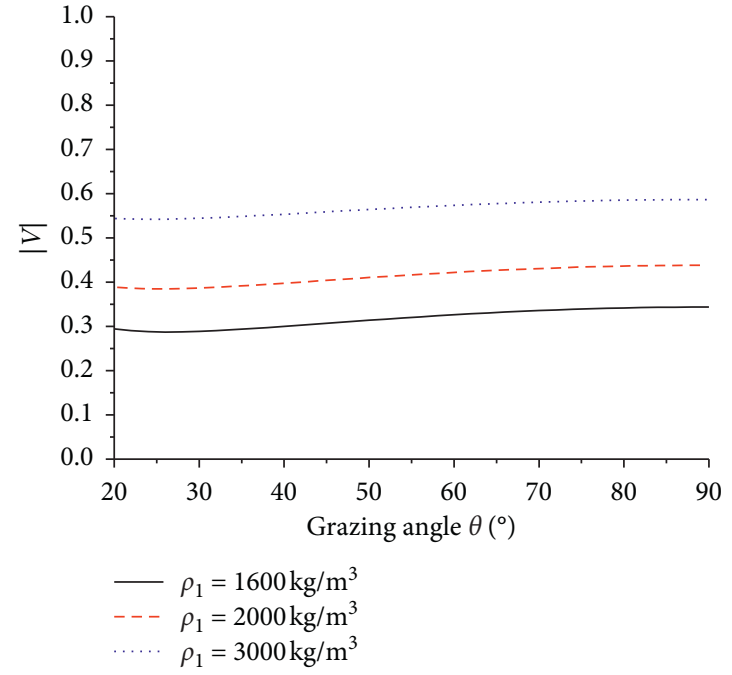

(d)

Figure 4: (a) The illustration of acoustic radiation with a boundary. (b) The $|V|-\theta$ curves with different compression wave speeds $\left(c_{s}=200 \mathrm{~m} / \mathrm{s}, \rho_{1}=1600 \mathrm{~kg} / \mathrm{m}^{3}\right)$. (c) The $|V|-\theta$ curves with different shear wave speeds $\left(c_{1}=1600 \mathrm{~m} / \mathrm{s}, \rho_{1}=1600 \mathrm{~kg} / \mathrm{m}^{3}\right)$. (d) The $|V|-\theta$ curves with different seabed densities $\left(c_{1}=1600 \mathrm{~m} / \mathrm{s}, c_{s}=200 \mathrm{~m} / \mathrm{s}\right)$.

TABle 1: The serial number $N_{S}$ of the different cases.

\begin{tabular}{ccccccccc}
\hline$N_{s}$ & 1 & 2 & 3 & 4 & 5 & 6 & 7 & 8 \\
\hline Case & $U_{0} D_{0}$ & $U_{1} D_{0}$ & $U_{1} D_{1}$ & $U_{2} D_{1}$ & $U_{2} D_{2}$ & $U_{3} D_{2}$ & $U_{3} D_{3}$ & $U_{4} D_{3}$ \\
\hline
\end{tabular}

3.2. The Study on the Effectiveness of the CWSM. According to the algorithms presented in Section 2, the process of the acoustic intensity vector field prediction of an elastic structure by using the CWSM could be concluded as follows:

(a) Establish the multiphysics field coupling numerical model by using the FEM for acquisition of the structural vibration. Meanwhile, the vibration information could also be provided with experimental measurement as the supplement.

(b) Compute the equivalent source intensities. Computational cost could be reduced with the approximations mentioned in Section 2.4.

(c) Select the appropriate computing model and the optimal transfer function. 


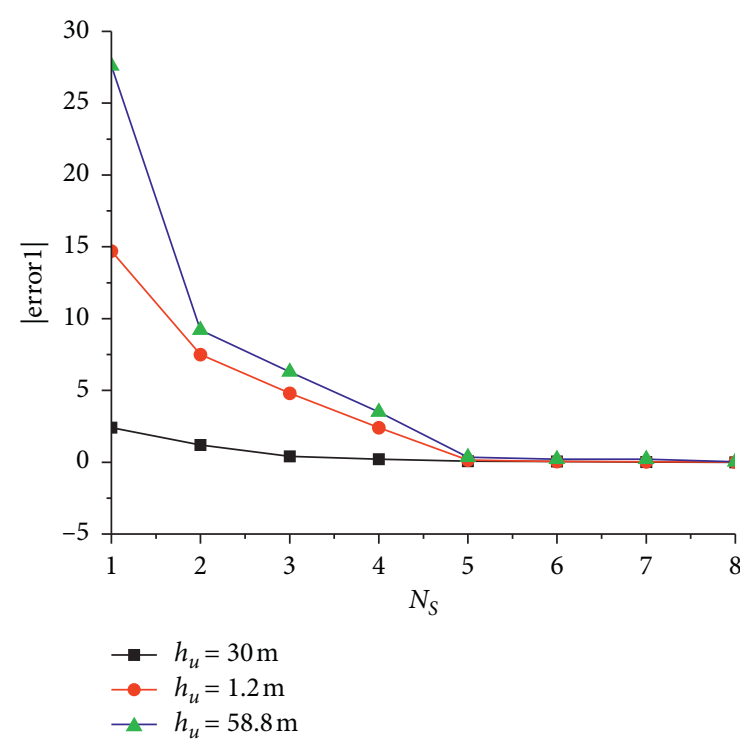

(a)

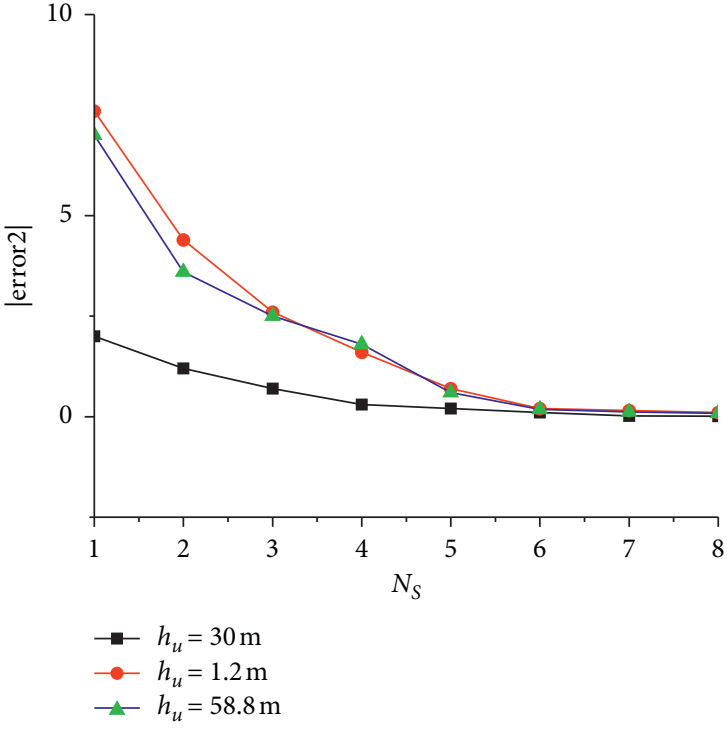

(b)

Figure 5: Average error ratios for the equivalent source intensities at different submerge depths $\left(f_{0}=120 \mathrm{~Hz}, c_{p}=1600 \mathrm{~m} / \mathrm{s}, c_{s}=200 \mathrm{~m} / \mathrm{s}\right.$, and $\rho_{1}=1600 \mathrm{~kg} / \mathrm{m}^{3}$ ).

(d) Compute the sound pressure and particle velocity in the waveguide with the WSM; then, the acoustic intensity could be obtained.

To verify the effectiveness of the CWSM to compute the acoustic intensity vector field of an elastic structure in a waveguide, the results of the finite element methodboundary element method (FEM-BEM) could be regarded as references since the transfer functions could be easily obtained. In the FEM-BEM, the acoustic and vibration responses on the structure surface are gained by the FEM and the computation of the sound field is accomplished by the BEM. The theoretical basis of the BEM is the Helmholtz boundary integral equation as below:

$$
C(P) p(P)=\int_{S}\left[g(P, \Lambda) \frac{\partial p(\Lambda)}{\partial \mathbf{n}_{\mathbf{s}}}-p(\Lambda) \frac{\partial g(P, \Lambda)}{\partial \mathbf{n}_{\mathbf{s}}}\right] \mathrm{d} S,
$$

where $S$ stands for the structural surface, $P$ represents points in the sound field, and $\Lambda$ represents integral points on the structural surface. $C(P)$ is a coefficient which relates to the position $P$ and when $P$ is in the sound field, $C(P)=1 . \mathbf{n}_{\mathbf{s}}$ is the normal vector on the integral point $\Lambda$, which points inwards the structure. $\partial p(\Lambda) / \partial \mathbf{n}_{\mathbf{s}}=-j \rho_{0} \omega_{0} v_{n}, v_{n}$ is the normal velocity on $\Lambda$.

It could be noted that the sound pressure and normal velocity on the structure surface are both necessary to be the acoustical inputs. Instead, the CWSM only regards the normal velocity on the structure surface as the acoustical input. Thus, the CWSM is more appropriate for actual engineering especially when the acoustical inputs are gained from measurements as the supplement for the FEM. Moreover, solving singular integral makes the computational cost of the FEM-BEM quite high that the method is not suitable for fast prediction.
According to Section 2.5, to compute the acoustic intensity vector field with a high accuracy, the complex sound pressure and the complex particle velocity in the sound field should be predicted precisely in advance. Suppose that the complex sound pressure $p_{c}$ could be written as $p_{c}=\widehat{p}+j \widetilde{p}$ and complex sound pressure $v_{c}$ could be written as $v_{c}=\widehat{v}+j \widetilde{v}$. Also, they could be expressed with other forms as $p_{c}=\left|p_{c}\right| \exp \left(j \gamma_{p}\right), v_{c}=\left|v_{c}\right| \exp \left(j \gamma_{v}\right), \gamma_{p}=\arctan (\tilde{p} / \tilde{p})$, and $\gamma_{v}=\arctan (\widetilde{v} / \widetilde{v})$. The sound pressure level is defined as SPL $=20 \log 10\left(|p| / p_{0}\right)$, where $p_{0}=10^{-6} \mathrm{~Pa}$. The particle velocity level is defined as $\mathrm{SVL}=20 \log 10\left(|v| / v_{0}\right)$, where $v_{0}=6.67 \times 10^{-13} \mathrm{~m} / \mathrm{s}$.

A shallow water waveguide with a liquid seabed is considered for simplicity. Suppose that the observation points are arranged 200 meters far from the center of the structure in the horizontal direction. The depths of the points are $0.5 \sim 59.5 \mathrm{~m}$ and the interval between two adjacent points is $1 \mathrm{~m}$.

The comparisons of the CWSM with the FEM-BEM are shown in Figures 7 and 8:

The computations are implemented on a computer, where the CPU is Intel(R) Core(TM) i7-4710MQ $(3.50 \mathrm{GHz})$ and the size of RAM is $16 \mathrm{~GB}$. The results of the CWSM and the FEM-BEM are in good agreement. According to the definition of complex acoustic intensity, if complex sound pressure $p_{c}$ and complex particle velocity $v_{c}$ could be acquired precisely, the acoustic intensity could also be computed with a high accuracy. Thus, the effectiveness of the CWSM for predicting the acoustic intensity vector field of an elastic structure in a waveguide could be verified. Meanwhile, combining equation (21) with equation (27), it could found that when computing the particle velocity in the sound field with the FEM-BEM, the second derivative of Green's function has to be solved with a high-computational cost which is evitable for the CWSM. For example, in the 
TABLE 2: Comparison for the modal frequencies and the mode shape of four modes around $120 \mathrm{~Hz}$ in free field, half-space, and a Pekeris waveguide.

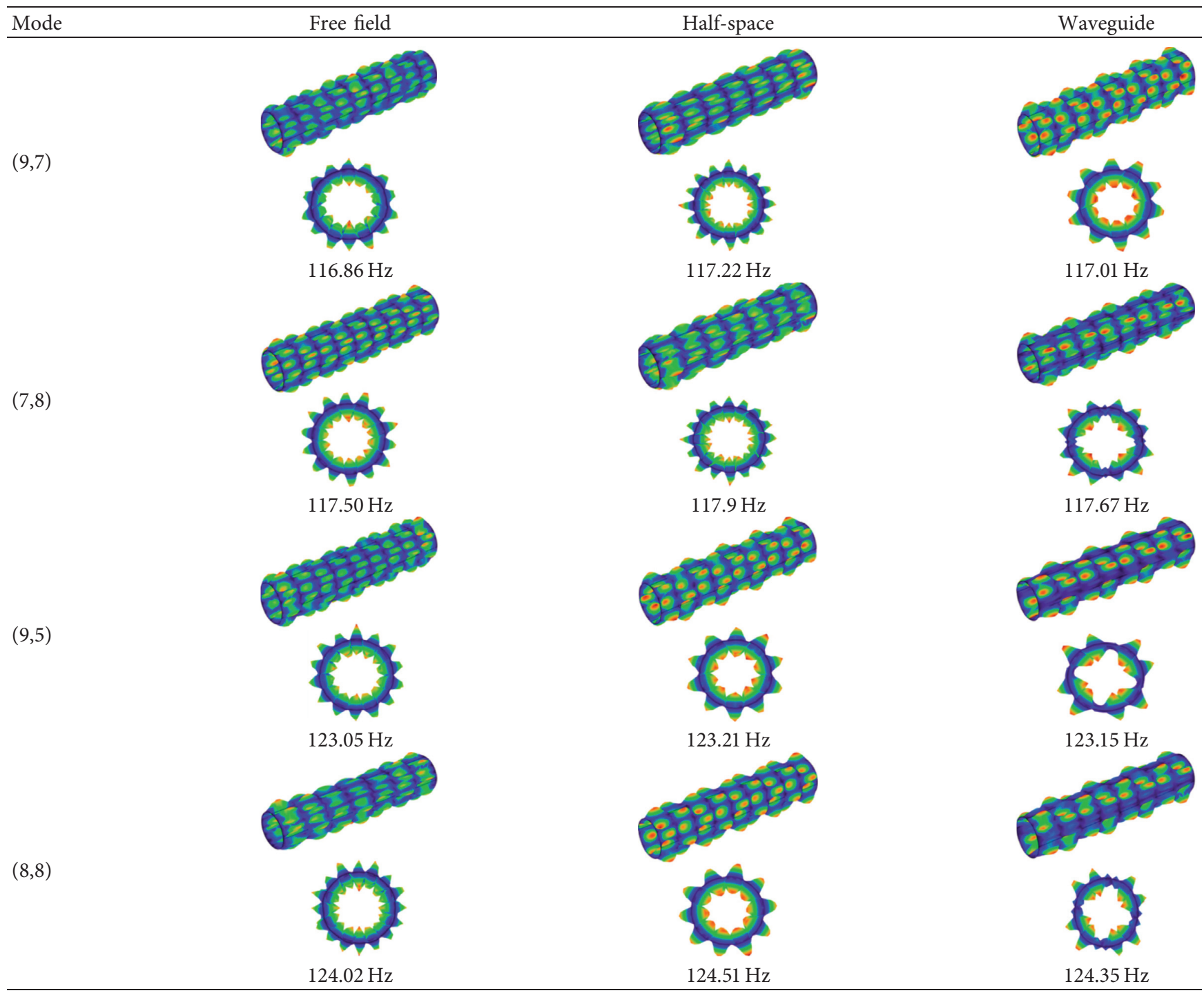

above simulations, it costs the FEM-BEM 1.8 minutes to accomplish the computation of the 60 field points. Instead, the CWSM only needs 0.6 minute. Therefore, compared with the FEM-BEM, the CWSM yields a higher computational speed and could be regarded as a prediction method with high efficiency.

3.3. Influence of the Source Type on the Sound Field. The sound radiation powers of the point source $W_{P}$ and the structure $W_{S}$ are

$$
\begin{aligned}
& W_{p}=\left.\frac{2 \pi r^{2} A^{2}}{\rho_{0} c_{0}}\right|_{r=1 m}, \\
& W_{S} \approx \frac{1}{2} \sum_{i=1}^{N} \operatorname{Re}\left(p \cdot v_{n}^{*}\right) \mathrm{d} S .
\end{aligned}
$$

According to equation (28), the pressure amplitude $A$ of the point source could be obtained. $A$ could be a coefficient to be put into equations (1) and (3) to make $W_{P}$ and $W_{S}$ equal.

In a Pekeris waveguide with a liquid seabed, the frequency for every mode is

$$
f_{n}=\frac{(2 n-1) \pi c_{0} c_{1}}{4 H \sqrt{c_{1}^{2}-c_{0}^{2}}}, \quad n=1,2 \ldots
$$

With the parameters given in Section 3.2, the normal mode frequencies in the waveguide are shown in Table 3:

When $f<f_{1}$, there are only evanescent waves which decay with the increase of range rapidly. Therefore, the compute frequency should not be lower than $f_{1}$.

When $f_{0}=50 \mathrm{~Hz}$, there is only one mode in the waveguide, as shown in Figure 9.

It is indicated that, at a frequency of $50 \mathrm{~Hz}$, the pressure distribution of a point source and a cylindrical shell tend to be similar at a long distance, as shown is Figure 9. 


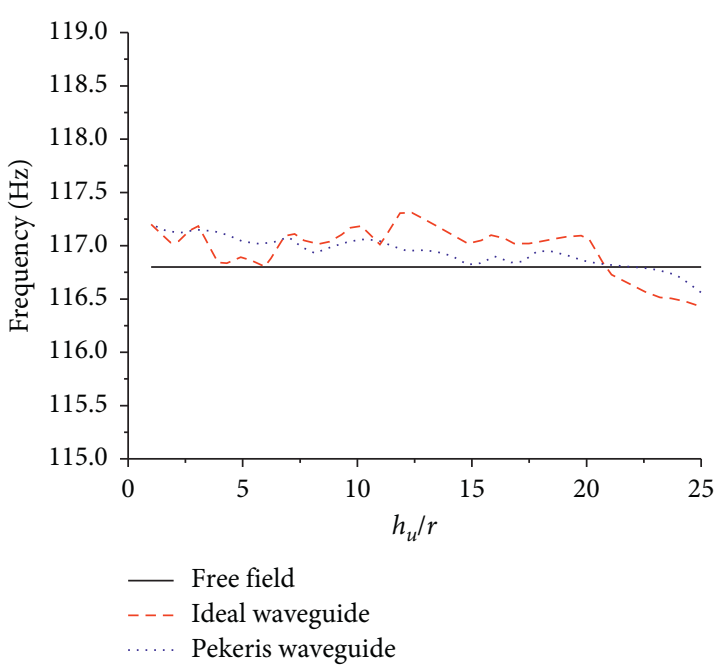

(a)

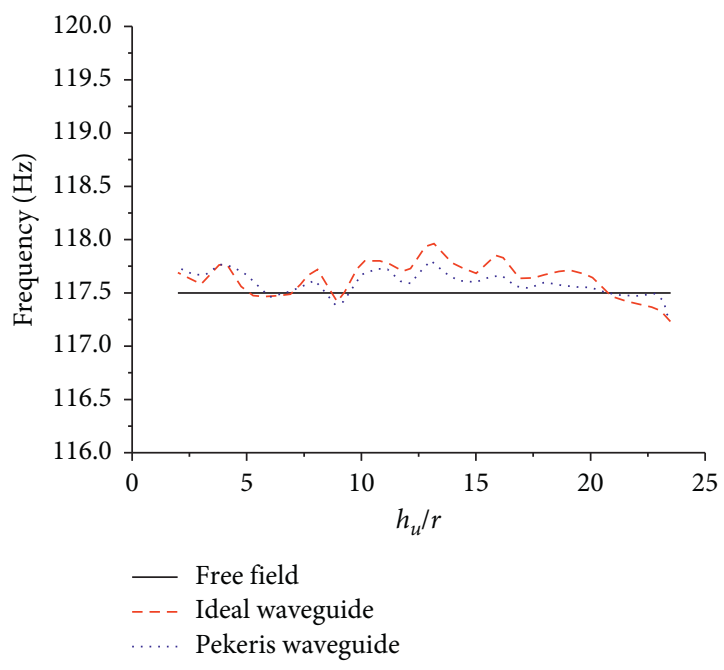

(c)

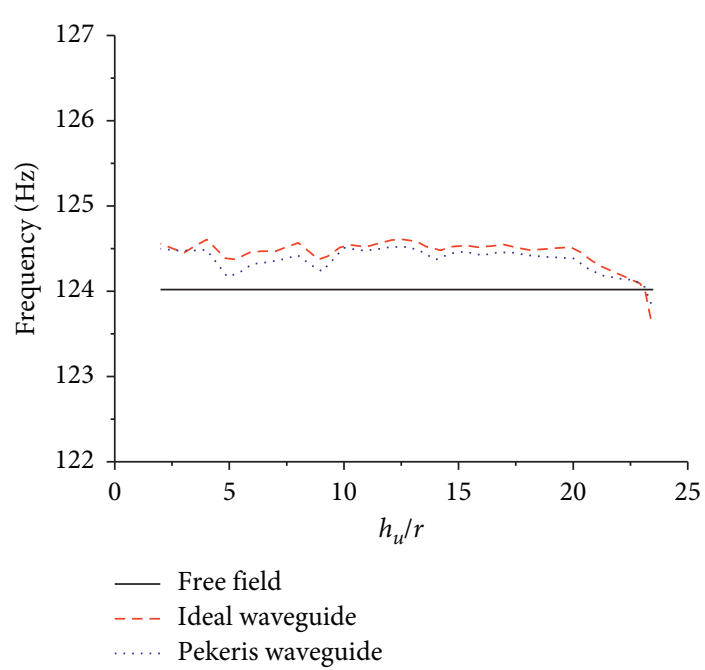

(b)

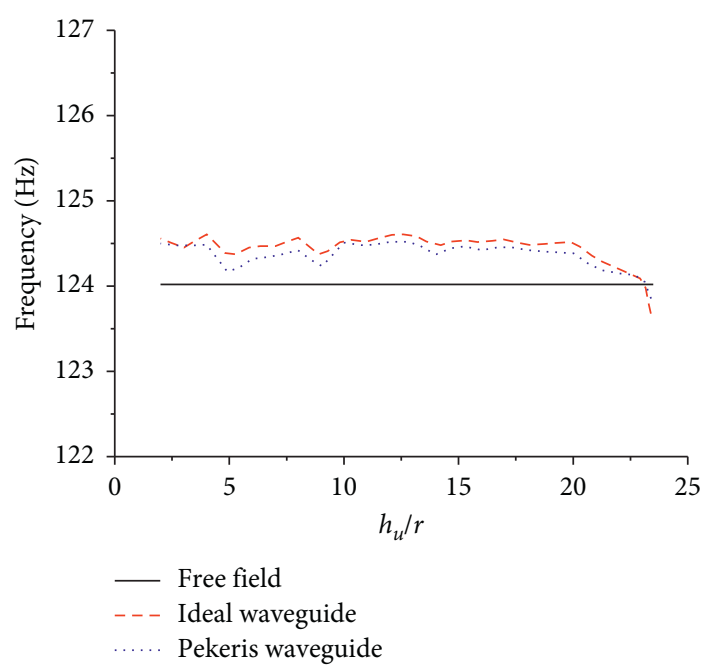

(d)

Figure 6: Modal frequency curves with different submerge depths. (a) $(9,7)$. (b) $(9,5)$. (c) $(7,8)$. (d) $(8,8)$.

The phenomenon in Figure 9 could be explained that, at a low frequency, the maximum linear dimension of the shell is much smaller than the wavelength and the vibration distribution is homogeneous, bringing in tiny phase diversities among different equivalent sources. Thus, without interference in the waveguide, there is no vortex in the acoustic intensity field. Moreover, since $\nabla\left|p^{2}\right| \propto Q, Q_{z}^{*}$ is positive when $\left|p^{2}\right|$ increases with $z$, and vice versa. $\left|I_{z}^{*}\right|$ tends to be zero at a long distance, which means that the sound energy mainly propagates along the horizontal direction.

However, at the near field, the sound field is not dominated by reflected sounds so that the normal mode theory is not suitable for computing the sound field. Instead, the sound field is mainly influenced by the direct sounds. As shown in Figure 10, for a structure, the vibration mode has a strong effect on the sound field near the source. Also, numerous interactions among the wavefronts from different equivalent sources contribute to more complicated spatial structure of the acoustic intensity field. Vortices appear in the acoustic intensity field for the structure, while the point source not.

Things would be different when considering a higher frequency. At the frequency of $190 \mathrm{~Hz}$, it turns to be a twomode case, as shown in Figure 11.

With the effect of destructive interference between the two modes, some vortices begin to appear in the acoustic intensity vector fields of the point source and the cylindrical shell. The overall trends of $\left|p^{2}\right|$ field for the two types of sources are still semblable. However, there are still some subtle differences. The black boxes in Figures 11(a) and 11(c) point out the dislocations at the depth between $25 \mathrm{~m}$ and $30 \mathrm{~m}$. The positions are $(845,27)$ and $(2535,26)$ for the point source. For the cylindrical shell, the positions are $(870,26)$ and $(2560,26)$. The discrepancies are derived from the amplitude and phase diversities among the equivalent sources for the shell. Moreover, according to David's work, for a two-mode case, the horizontal interval between two adjacent dislocations, $\Delta D$, depends on the eigenvalues in the 


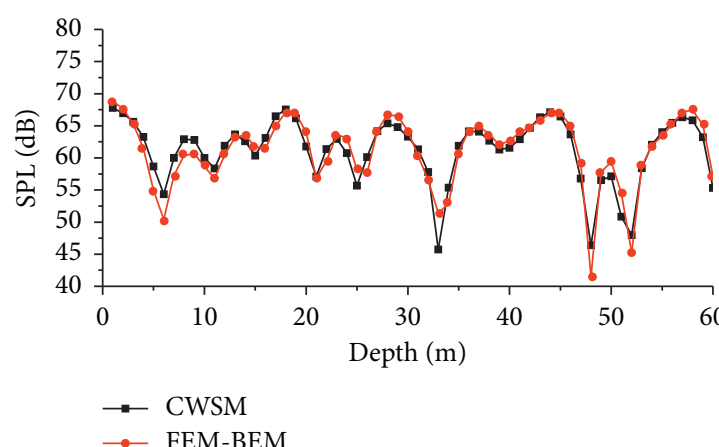

(a)

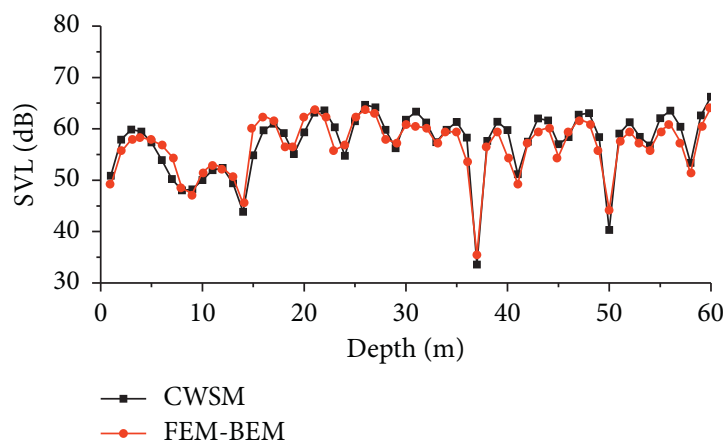

(c)

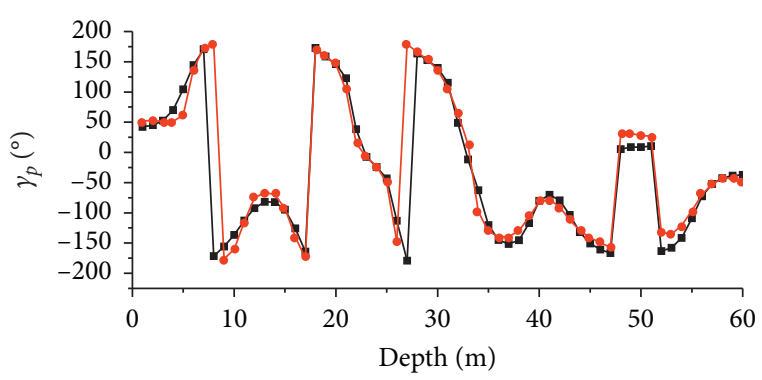

- CWSM

$\because$ FEM-BEM

(b)

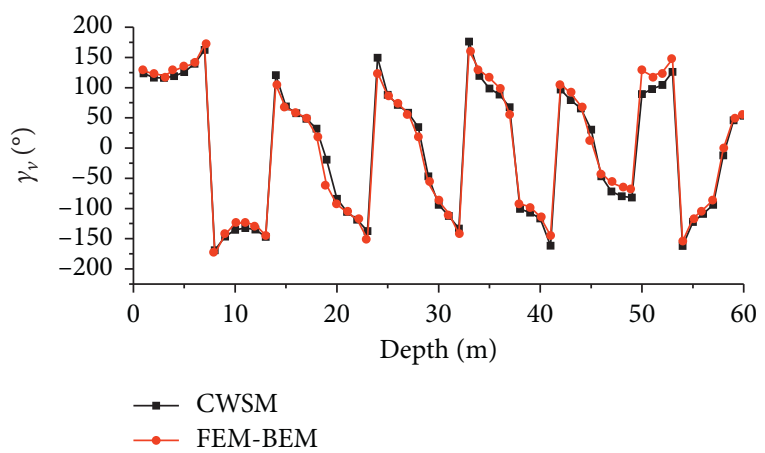

(d)

FIGURe 7: Comparisons of the CWSM with the FEM-BEM at the frequency of $190 \mathrm{~Hz}$. (a) SPL. (b) $\gamma_{p}$. (c) SVL. (d) $\gamma_{v}\left(c_{1}=1520 \mathrm{~m} / \mathrm{s}\right.$ and $\left.\rho_{1}=1600 \mathrm{~kg} / \mathrm{m}^{3}\right)$.

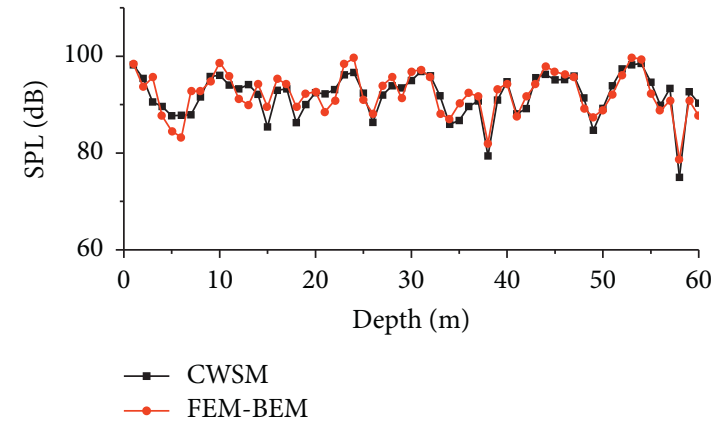

(a)

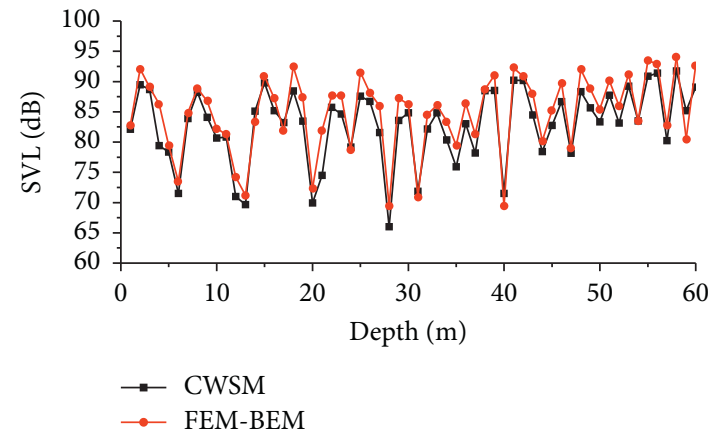

(c)

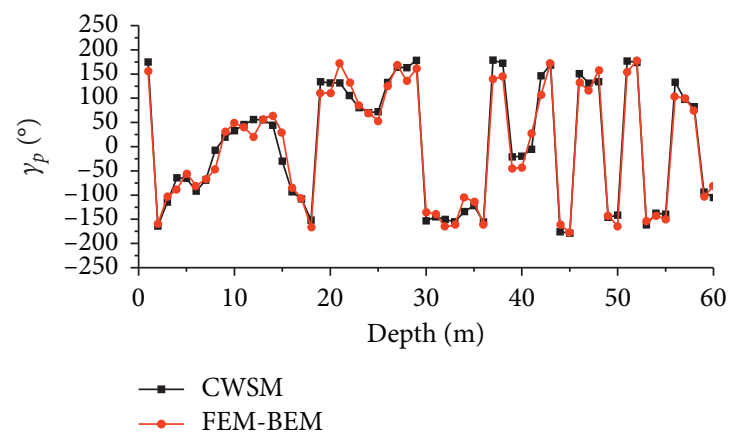

(b)

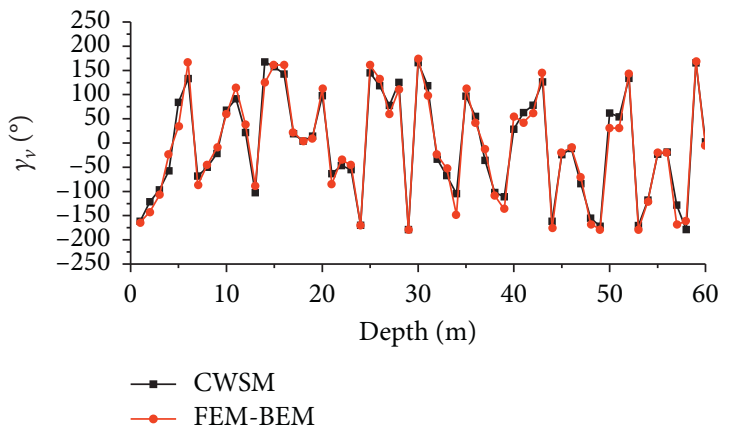

(d)

Figure 8: Comparisons of the CWSM with the FEM-BEM at the frequency of $360 \mathrm{~Hz}$. (a) SPL. (b) $\gamma_{p}$. (c) SVL. (d) $\gamma_{v}\left(c_{1}=1520 \mathrm{~m} / \mathrm{s}\right.$ and $\rho_{1}=1600 \mathrm{~kg} / \mathrm{m}^{3}$ ). 
TABLE 3: Normal mode frequencies in the waveguide.

\begin{tabular}{lccccc}
\hline$n$ & 1 & 2 & 3 & 4 & 5 \\
\hline$f_{n} / \mathrm{Hz}$ & 38.66 & 115.96 & 193.27 & 270.58 & 347.89 \\
\hline
\end{tabular}

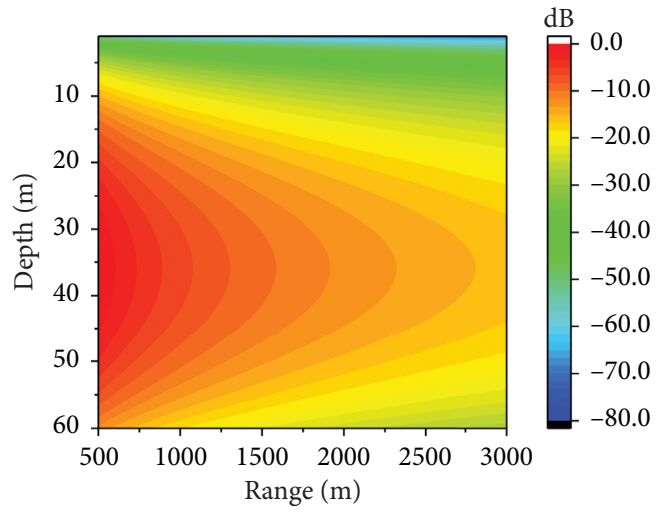

(a)

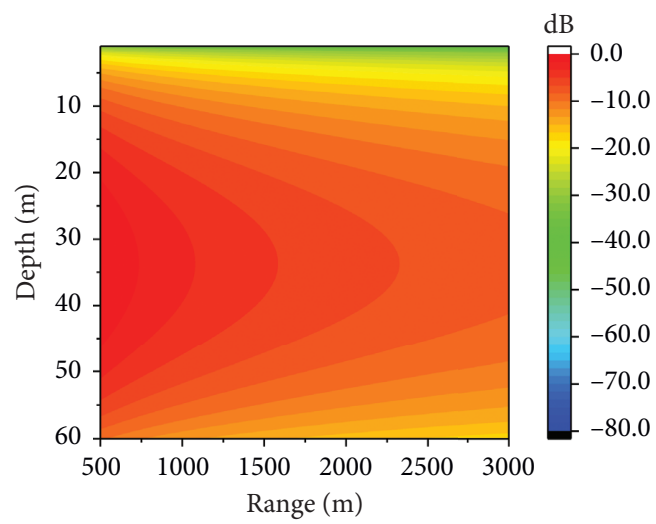

(c)
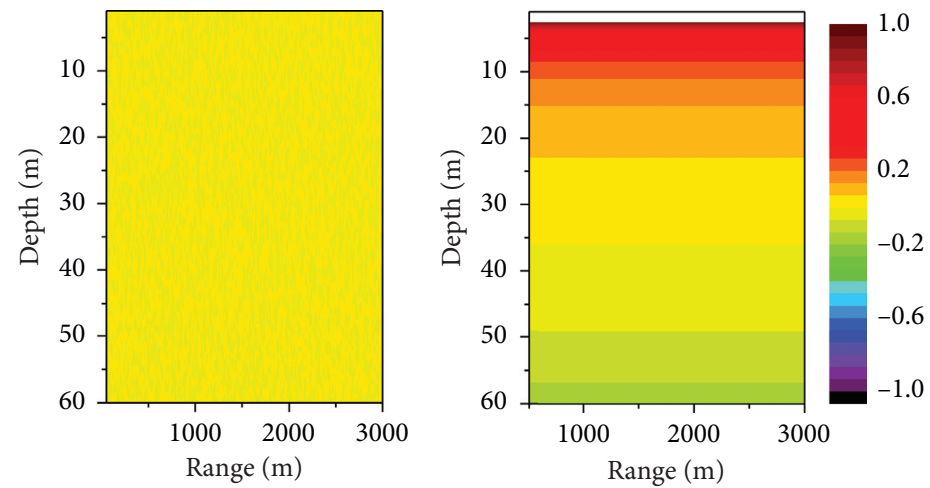

(b)
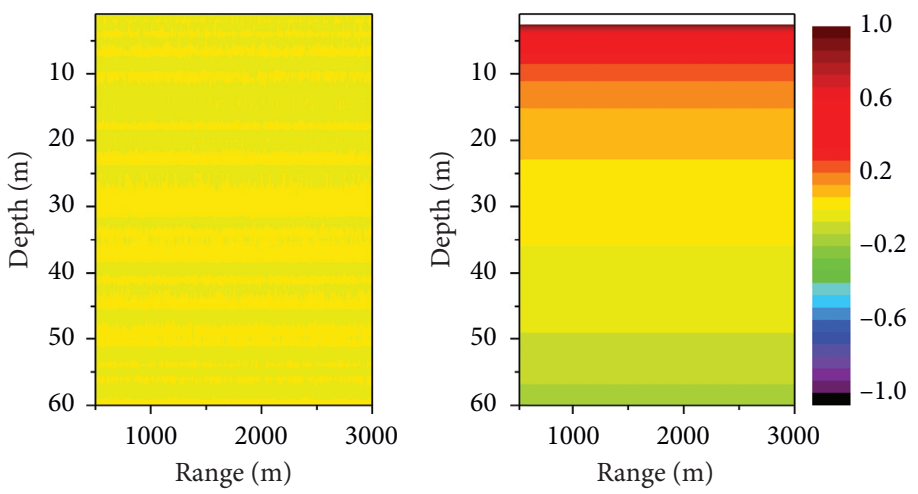

(d)

FIGURE 9: (a) Normalized $\left|p^{2}\right|$ field of a unit point source. (b) Normalized acoustic intensity field of the unit point source (left: $I_{z}^{*}$; right: $Q_{z}^{*}$ ). (c) Normalized $\left|p^{2}\right|$ field of a cylindrical shell. (d) Normalized acoustic intensity field of the cylindrical shell (left: $I_{z}^{*} ;$ right: $\left.Q_{z}^{*}\right)\left(h_{U}=20 \mathrm{~m}\right.$, $\left.f_{0}=50 \mathrm{~Hz}\right)$.
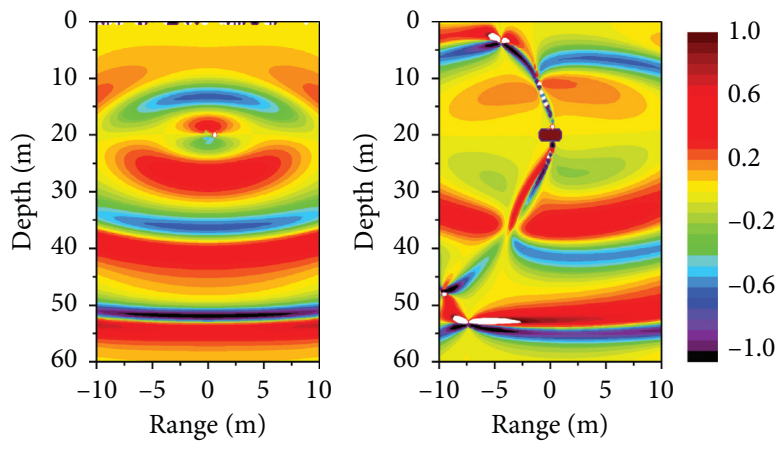

(a)
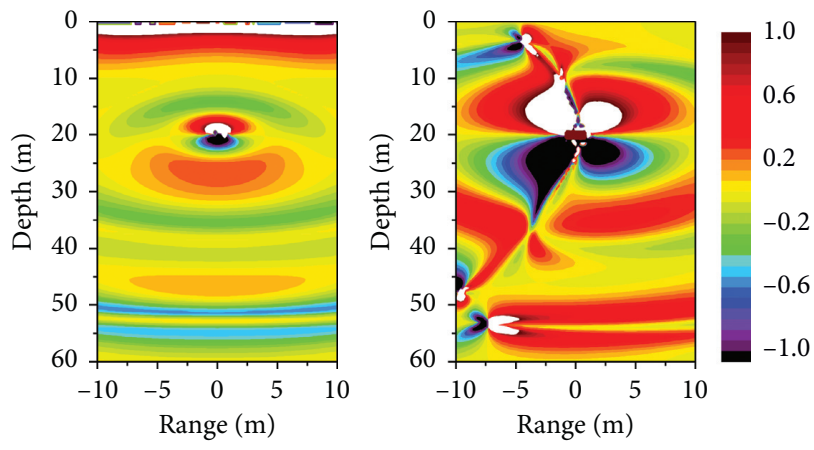

(b)

FIgURe 10: (a) $I_{z}^{*}$ near the source (range from $-10 \mathrm{~m}$ to $10 \mathrm{~m}$; left: point source; right: cylindrical shell). (b) $Q_{z}^{*}$ near the source (range from $-10 \mathrm{~m}$ to $10 \mathrm{~m}$. left: point source; right: cylindrical shell) $\left(f_{0}=50 \mathrm{~Hz}\right.$ and $\left.h_{U}=20 \mathrm{~m}\right)$. 


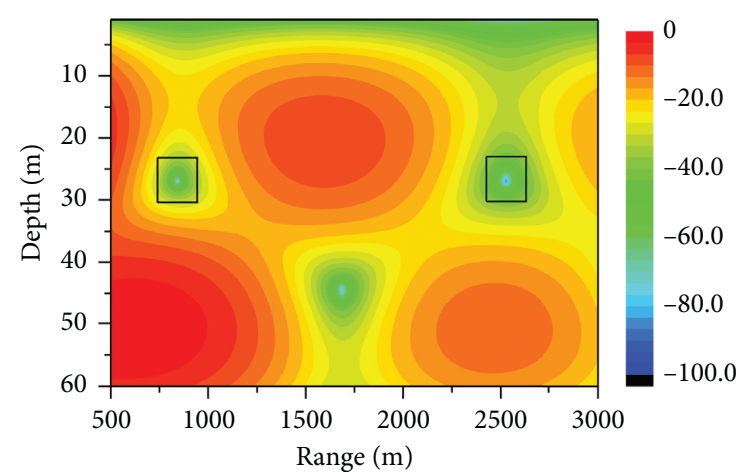

(a)

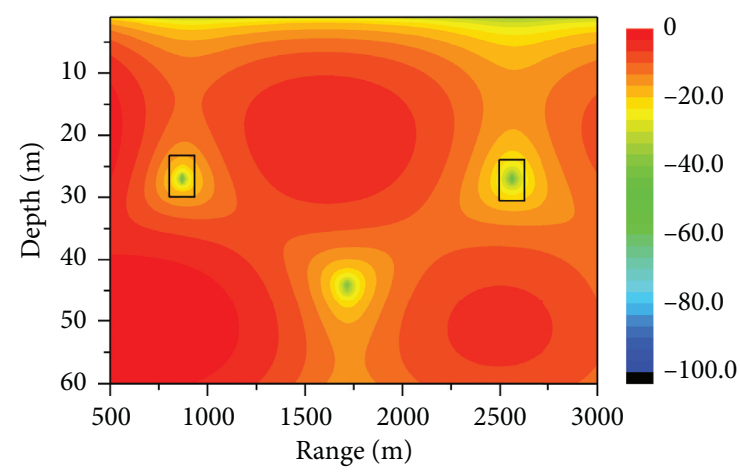

(c)
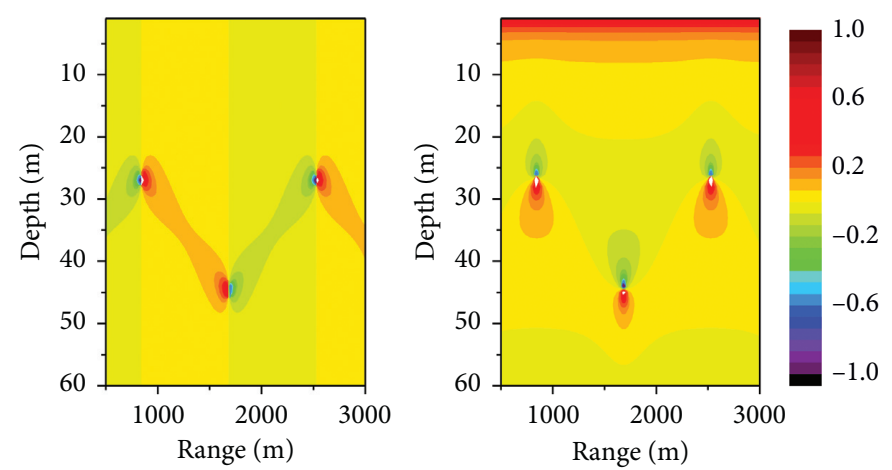

(b)
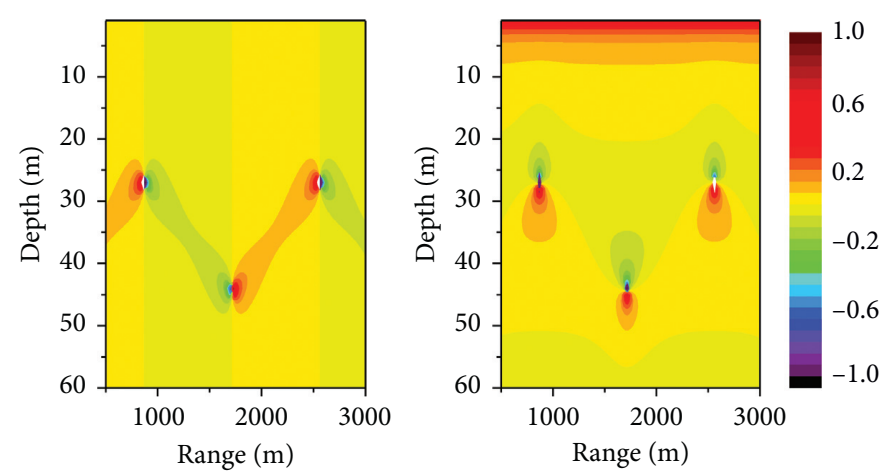

(d)

FIGURE 11: (a) Normalized $\left|p^{2}\right|$ field of a unit point source. (b) Normalized acoustic intensity field of the unit point source (left: $I_{z}^{*}$; right: $Q_{z}^{*}$ ). (c) Normalized $\left|p^{2}\right|$ field of the cylindrical shell. (d) Normalized acoustic intensity field of the cylindrical shell (left: $I_{z}^{*}$; right: $Q_{z}^{*}$ ); $\left(h_{U}=20 \mathrm{~m}\right.$ and $\left.f_{0}=190 \mathrm{~Hz}\right)$.

Pekeris waveguide [32]. In the above simulation, $\Delta D=2 \pi /\left(\xi_{1}-\xi_{2}\right)$ is satisfied for both the point source and the cylindrical shell since the horizontal eigenvalues for a waveguide are invariable at a fixed frequency.

Meanwhile, as shown in Figure 10, the behaviors of $I_{z}^{*}$ along the horizontal direction are opposite, which implies that the dynamic characteristics of sound energy at the vortex regions are entirely different for the two types of sources, which provides a profound understanding of acoustic propagation in waveguides. The key advantage of the normalized acoustic intensity is that the absolute values of the active and reactive components are maximum at dislocations and minimum at crests in the acoustic intensity vector field. Detection of these regions is convenient by a vertical line array. With the analysis of the acoustic intensity field, not only the distribution but also the dynamic characteristics of the sound field could be obtained.

Furthermore, if the frequency is higher, at the frequency of $360 \mathrm{~Hz}$, it is a five-mode case, as shown in Figure 12.

From the above simulations, it could be concluded that the interference effects could be more complicated with more normal modes in the waveguide, which could bring more vortices into the acoustic intensity field. According to equation (29), the total mode quantity in a Pekeris waveguide with a liquid seabed could be written as follows:

$$
N_{m}=\frac{2 f_{0} H \sqrt{c_{1}^{2}-c_{0}^{2}}}{\pi c_{0} c_{1}}+\frac{1}{2} .
$$

From equation (30), $N_{m}$ mainly depends on the following factors: the waveguide depth $H$, the compute frequency $f_{0}$, and the sound speed in the seabed $c_{1}$. It has been analyzed that, with a higher $f_{0}, N_{m}$ is larger, bringing in more complicated interferences in the waveguide and more vortices generate. In a similar way, it could be concluded that, with the increase of $H$ and $c_{1}$, there would be a larger $N_{m}$, leading to the same phenomenon.

As shown in Figures 13 and 14, for the sound field near the source, there are still outstanding discrepancies for the two types of sources. The vibration modes of the cylindrical shell make the spatial structure of sound field more complicated. Therefore, the positions of the vortices are entirely different for the two kinds of sources. With the increase of the frequency, the order of vibration mode becomes higher and the effect of the direct sound is stronger, causing the phenomenon more obvious.

To make the analyses concise and intuitive, a waveguide with a liquid seabed is considered in the above simulations. Actually, with shear waves in the seabed, only reflection coefficient and attenuation coefficient are affected among the parameters, and normal modes could also form in the waveguide. Therefore, the spatial structure in the acoustic 


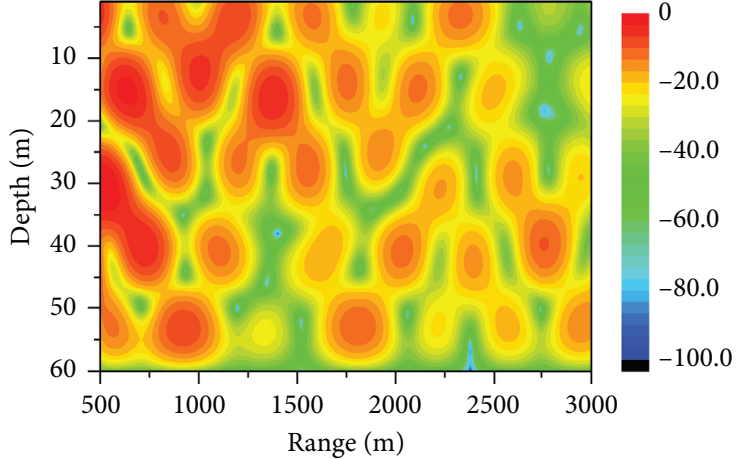

(a)

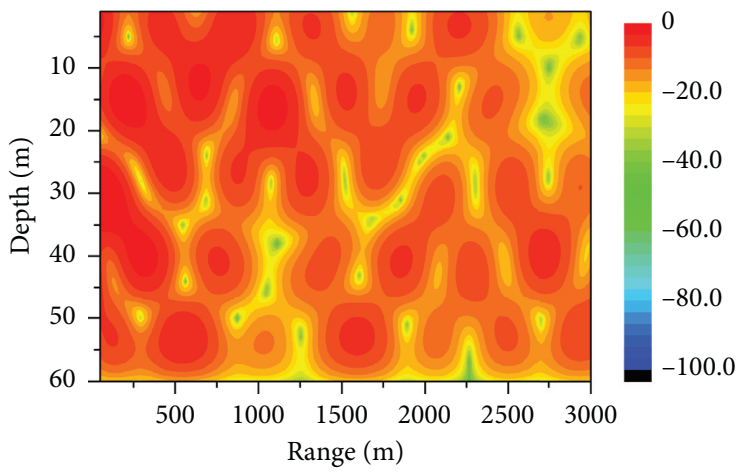

(c)
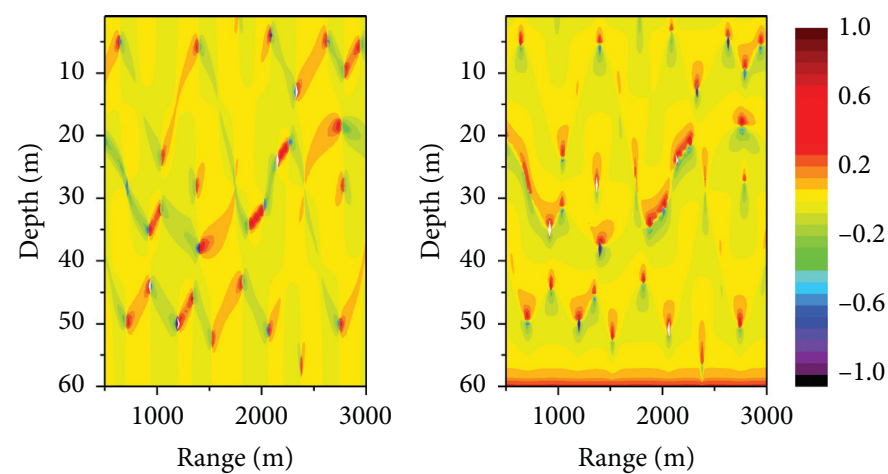

(b)
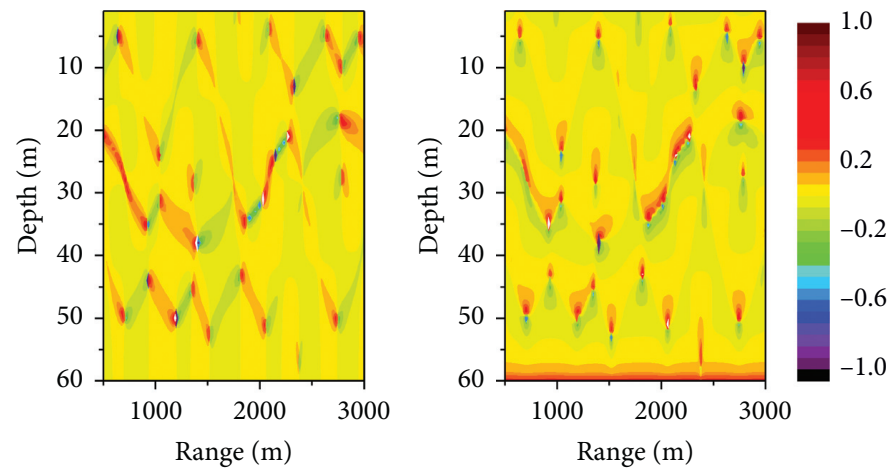

(d)

Figure 12: (a) Normalized $\left|p^{2}\right|$ field of a unit point source. (b) Normalized acoustic intensity field of the point source (left: $I_{z}^{*} ;$ right: $Q_{z}^{*}$ ). (c) Normalized $\left|p^{2}\right|$ field of the cylindrical shell. (d) Normalized acoustic intensity field of the cylindrical shell (left: $I_{z}^{*}$; right: $Q_{z}^{*}$ ) $\left(h_{U}=20 \mathrm{~m}\right.$ and $\left.f_{0}=360 \mathrm{~Hz}\right)$.
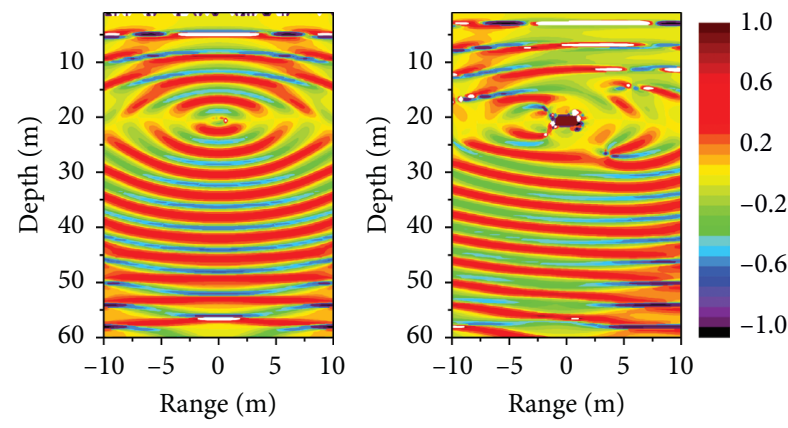

(a)
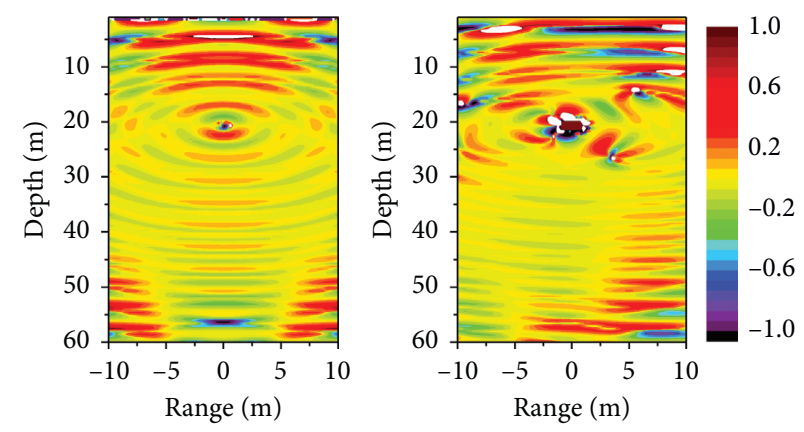

(b)

Figure 13: (a) $I_{z}^{*}$ near the source (range from $-10 \mathrm{~m}$ to $10 \mathrm{~m}$; left: point source; right: cylindrical shell). (b) $Q_{z}^{*}$ near the source (range from $-10 \mathrm{~m}$ to $10 \mathrm{~m}$. left: point source; right: cylindrical shell $)\left(f_{0}=190 \mathrm{~Hz}\right.$ and $\left.h_{U}=20 \mathrm{~m}\right)$.

intensity field could also be explained by the normal mode theory and the ray theory.

3.4. The Acoustic Intensity Vector Field in a Waveguide with Shear Waves in the Seabed. In a waveguide with a liquid seabed, the normal mode quantity could be calculated easily with equation (30). In practice, the eigenvalues of the normal modes are difficult to be expressed analytically in a waveguide with shear waves in the seabed. In this paper, the mode quantity and eigenvalues are computed with Kraken C, which is a sophisticated algorithm for computing the eigenvalues in the waveguide with shear waves in the seabed. Many factors may have impacts on the acoustic intensity vector field, such as the seismic wave speeds in the seabed, the density of the seabed, the compute frequency, and the submerge depth. The influences of the factors will be analyzed in the following part.

The vortices in the acoustic intensity field are derived from the interferences among different normal modes. According to Zhu's work [33], the interference properties could be better described by active vertical intensity $I_{z}$ since it only includes interference terms: 

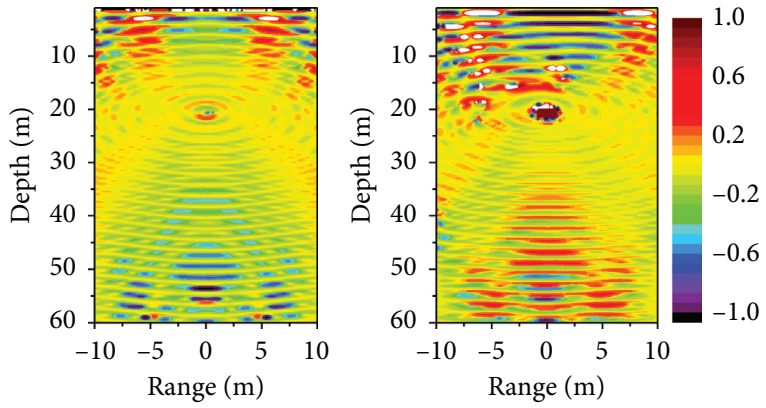

(a)
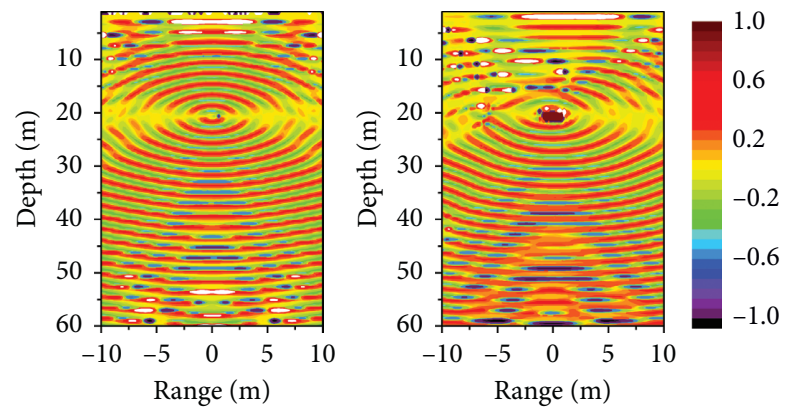

(b)

Figure 14: (a) $I_{z}^{*}$ near the source (range from $-10 \mathrm{~m}$ to $10 \mathrm{~m}$; left: point source; right: cylindrical shell). (b) $Q_{z}^{*}$ near the source(range from $-10 \mathrm{~m}$ to $10 \mathrm{~m}$. left: point source; right: cylindrical shell $)\left(f_{0}=360 \mathrm{~Hz}\right)$.

$$
\operatorname{Re}\left[I_{z}(r, z)\right] \approx \frac{1}{\rho_{1} \omega_{0}} \sum_{n, n \neq \varepsilon}^{N} \sum_{\varepsilon=1}^{N}\left[\frac{\Phi_{\varepsilon}^{\prime}(z)}{\Phi_{\varepsilon}(z)}\right]^{*} \Psi_{n} \Psi_{\varepsilon} \sin \left(\Delta \xi_{\varepsilon n} r\right)
$$

where $\Phi_{\varepsilon}(z)$ is the eigenfunction of $z . \Psi_{n}$ is the pressure modal amplitude which could be expressed as follows:

$$
\Psi_{n}=j 2 \pi \Phi_{n}(z) \Phi_{n}\left(z_{0}\right) H_{0}^{(2)}\left(\xi_{n} r\right) .
$$

Thus, only $I_{z}^{*}$ is investigated in the following part for analyzing the dynamic characteristic of the sound energy along the vertical direction. It must be pointed out that the compression wave speed $c_{1}$ and the shear wave speed $c_{s}$ satisfy a fixed mathematic relation with a certain material of the seabed, as shown in the appendix. In the paper, three simulation models are defined with different wave speeds in the seabed as follows (Table 4).

Firstly, the effect of shear waves in the seabed is analyzed in Figure 15:

It could be seen that, with a liquid seabed, the fluctuation of sound energy is more obvious than that with an elastic seabed. In addition, with the increase of $c_{s}$, the absolute value of $I_{z}^{*}$ drops slightly, which means that, with the comsumption of the shear waves, the high-order modes attenuate more rapidly. Thus, interferences caused by reflected sound could be weaker, especially at a long distance.

It could be seen in Figure 16 that $N_{m}$ for the three models are 2,3 , and 4 , respectively. With the consumption of shear waves, high-order modes attenuate very fast in the horizontal direction. When $r$ is large enough, there may be only the first mode and no vortex exist in the waveguide. Generally, the normal modes attenuate more rapidly along the $r$-axis with the increase of order. However, in Model III, the attenuation coefficients of the first and the fourth modes are much smaller than the others. Thus, the vortices far from the source derive from the interference between the first and the fourth modes. The horizontal wave number $\xi_{n}$ for Model III could be shown in Table 5 when $f_{0}=50 \mathrm{~Hz}$.

For the normal modes which could propagate in the water layer, it should satisfy $k_{1}<\xi_{n}<k_{0}$. When $\xi_{n}>k_{0}$, the mode represents Scholte surface wave. When $\xi_{n}<k_{1}$, the modes are regarded as "leaky modes," which attenuate
TABle 4: Simulation models with different wave speeds in the seabed (unit: $\mathrm{m} / \mathrm{s}$ ).

\begin{tabular}{lcc}
\hline Model & $c_{1}$ & $c_{s}$ \\
\hline I & 1600 & 200 \\
II & 1800 & 800 \\
III & 2000 & 1200 \\
\hline
\end{tabular}

exponentially in the horizontal direction. The imaginary part of $\xi_{n}$ is the modal attenuation coefficient of the $n$th mode.

In Model III, $k_{1}=0.1571$. It could be found that the $\xi_{4}$ is very close to $k_{1}$. In Zhang's work, he found that when a mode is very close to "leaky modes," the modal attenuation coefficient could be very small, making the mode attenuate along the horizontal direction quite slowly [24]. Therefore, even a high-order mode could propagate far in this situation.

The influence of source frequency is analyzed in Figure 17. Obviously, with the increase of $f_{0}, N_{m}$ becomes larger. Thus, more vortices exist in the waveguide since the interferences are more complicated.

When the density of the seabed varies, $N_{m}$ always remains a constant. Instead, only the amplitudes of every mode have minor variations. From Figure 18, it could be found that $\rho_{1}$ does not significantly influence the acoustic intensity vector field, especially at a long distance.

According to Figure 19, though $N_{m}$ is a constant for different submerge depths of the cylindrical shell, the behaviors of $I_{z}^{*}$ still manifest variously. When $h_{U}=30 \mathrm{~m}$, the source is close to the center depth of the waveguide and the sound energy distribution in the waveguide is more homogeneous with few vortices in the waveguide. However, when $h_{U}=5 \mathrm{~m}$ or $h_{U}=55 \mathrm{~m}$, since the effect of reflection is stronger when the source is more close to the boundaries, the sound energy fluctuates more obviously and more vortices appear in the acoustic intensity field.

With the increase of $H, N_{m}$ is becoming larger. In Figure 20, the $N_{m}$ are 4, 5, and 10 for the three waveguide depths, respectively. However, for different waveguide depths, the attenuation coefficient of every mode could be entirely different. When $H=60 \mathrm{~m}$, the attenuation coefficient of the 4th mode is even less than that of the first mode. 


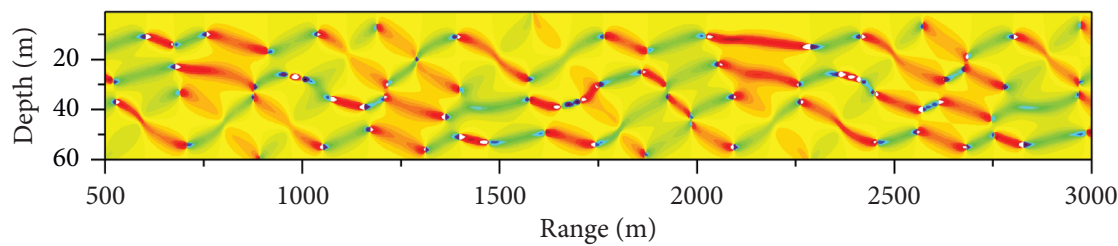

(a)

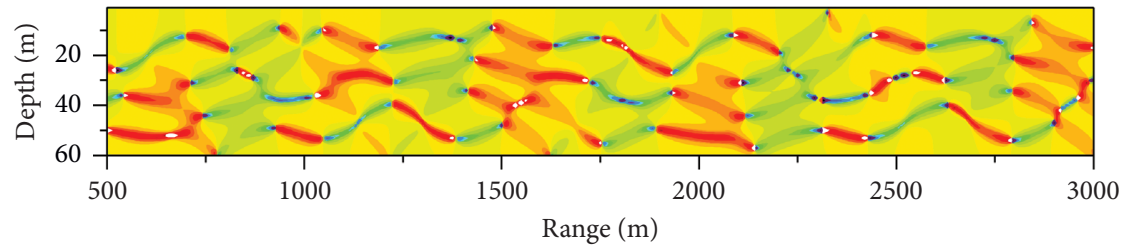

(b)

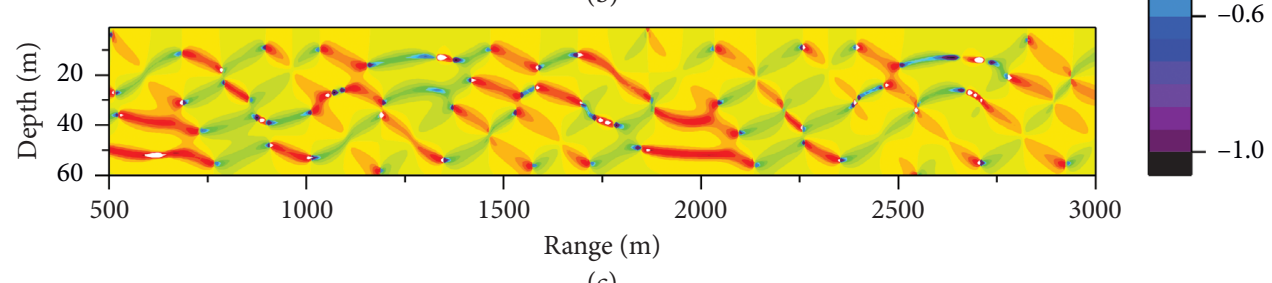

(c)

Figure 15: $I_{z}^{*}$ for different seabed types when $f_{0}=190 \mathrm{~Hz}, \rho_{1}=1600 \mathrm{~kg} / \mathrm{m}^{3}, h_{U}=20 \mathrm{~m}$, and $c_{1}=1600 \mathrm{~m} / \mathrm{s}$ ((a) liquid seabed; (b) $c_{s}=200 \mathrm{~m} / \mathrm{s} ;$ (c) $\left.c_{s}=600 \mathrm{~m} / \mathrm{s}\right)$.

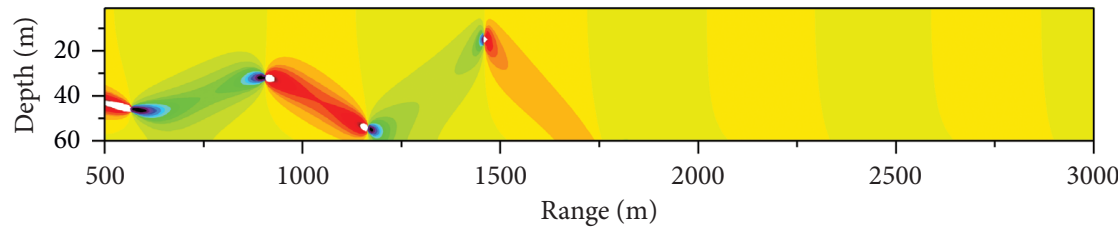

(a)

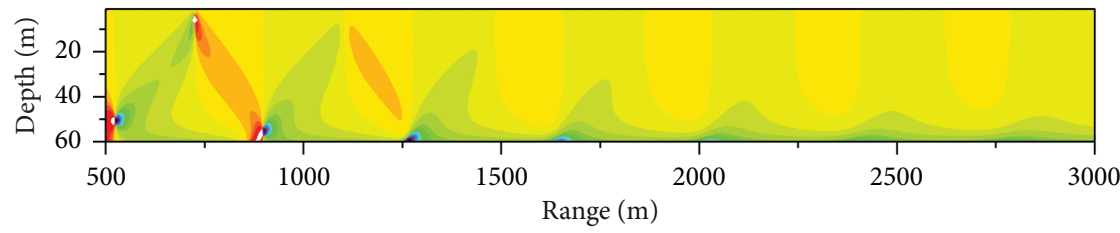

(b)
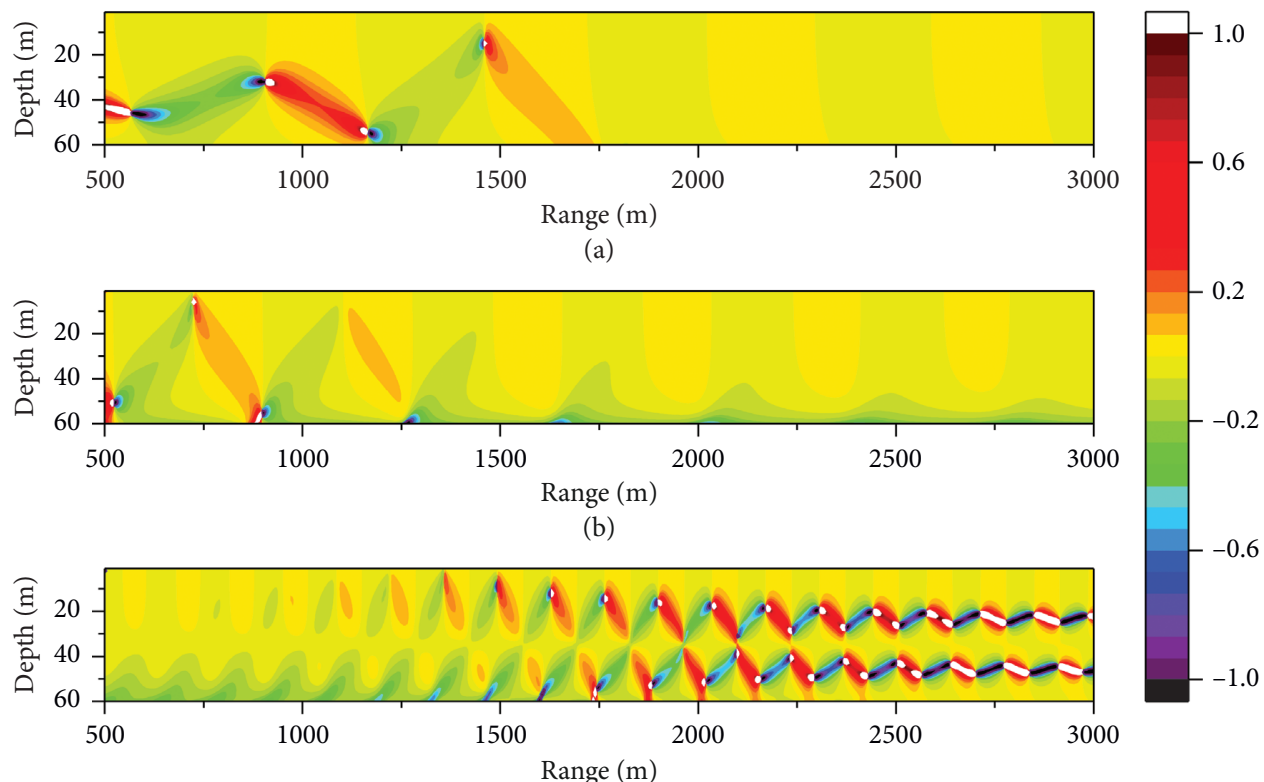

(c)

Figure 16: $I_{z}^{*}$ for different seismic wave speeds when $f_{0}=50 \mathrm{~Hz}, \rho_{1}=1600 \mathrm{~kg} / \mathrm{m}^{3}$, and $h_{U}=20 \mathrm{~m}$ ((a) Model I; (b) Model II; (c) Model III).

TABLe 5: $\xi_{n}$ for Model III $\left(f_{0}=50 \mathrm{~Hz}\right)$.

\begin{tabular}{lc}
\hline Mode & $\xi_{n}$ \\
\hline 1st & $0.20352+0.00204 i$ \\
2nd & $0.18656+0.00154 i$ \\
3rd & $0.17426+0.02316 i$ \\
4th & $0.15722+0.00041 i$ \\
5th & $0.10206+0.01840 i$ \\
\hline
\end{tabular}

Therefore, the fourth mode could even exist at a very long distance to create vortices. However, when $H=120 \mathrm{~m}$ and $H=240 \mathrm{~m}$, the modal attenuation coefficients increase with the increase of the order, meaning that only few low-order modes which could hardly create vortices exist at a long distance. It could be seen that, though there are numerous normal modes in the waveguide, the high-order modes attenuate quite rapidly and the effect of interferences is likely to be weak at a long distance. 


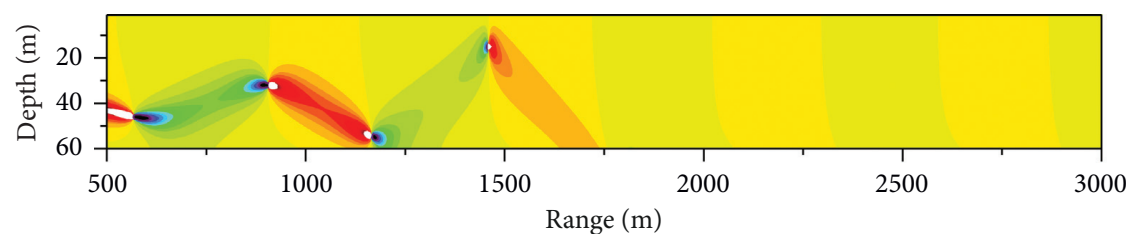

(a)

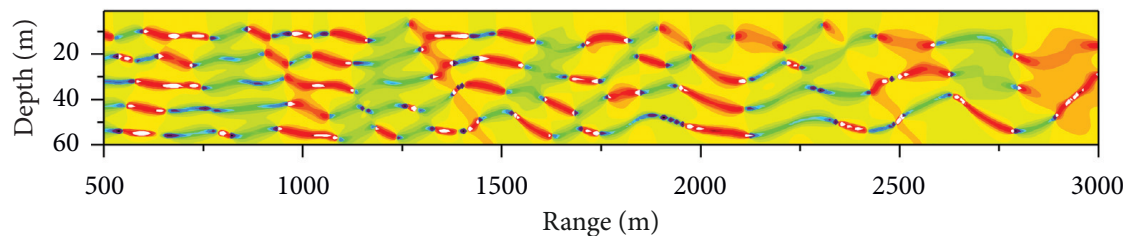

(b)

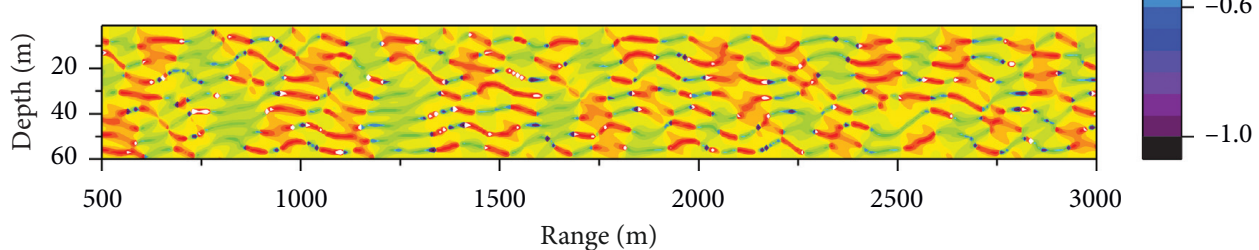

(c)

Figure 17: $I_{z}^{*}$ for different compute frequencies when $\rho_{1}=1600 \mathrm{~kg} / \mathrm{m}^{3}, h_{U}=20 \mathrm{~m}, c_{1}=1600 \mathrm{~m} / \mathrm{s}$, and $c_{s}=200 \mathrm{~m} / \mathrm{s}\left((\mathrm{a}) f_{0}=50 \mathrm{~Hz}\right.$; (b) $f_{0}=190 \mathrm{~Hz} ;(\mathrm{c}) f_{0}=360 \mathrm{~Hz}$ ).

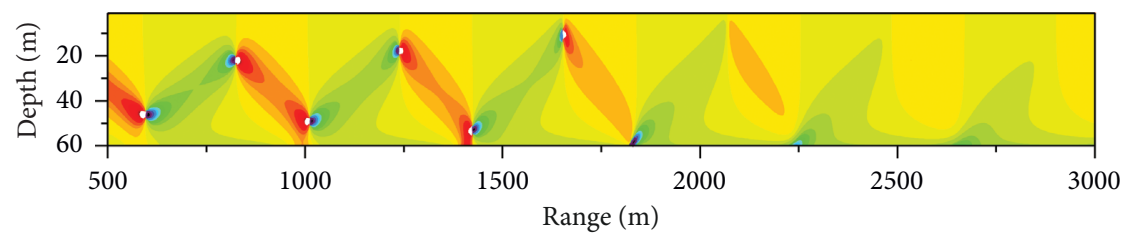

(a)

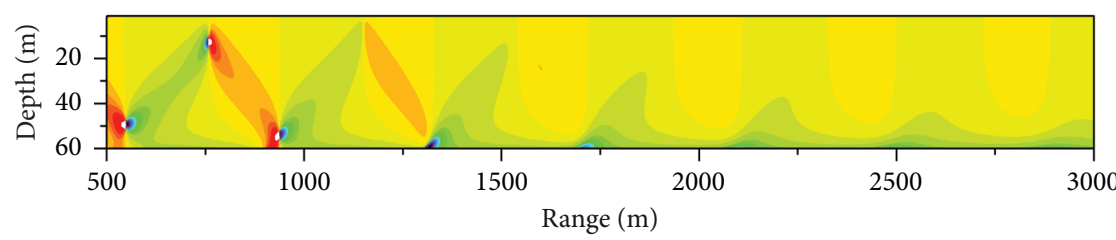

(b)
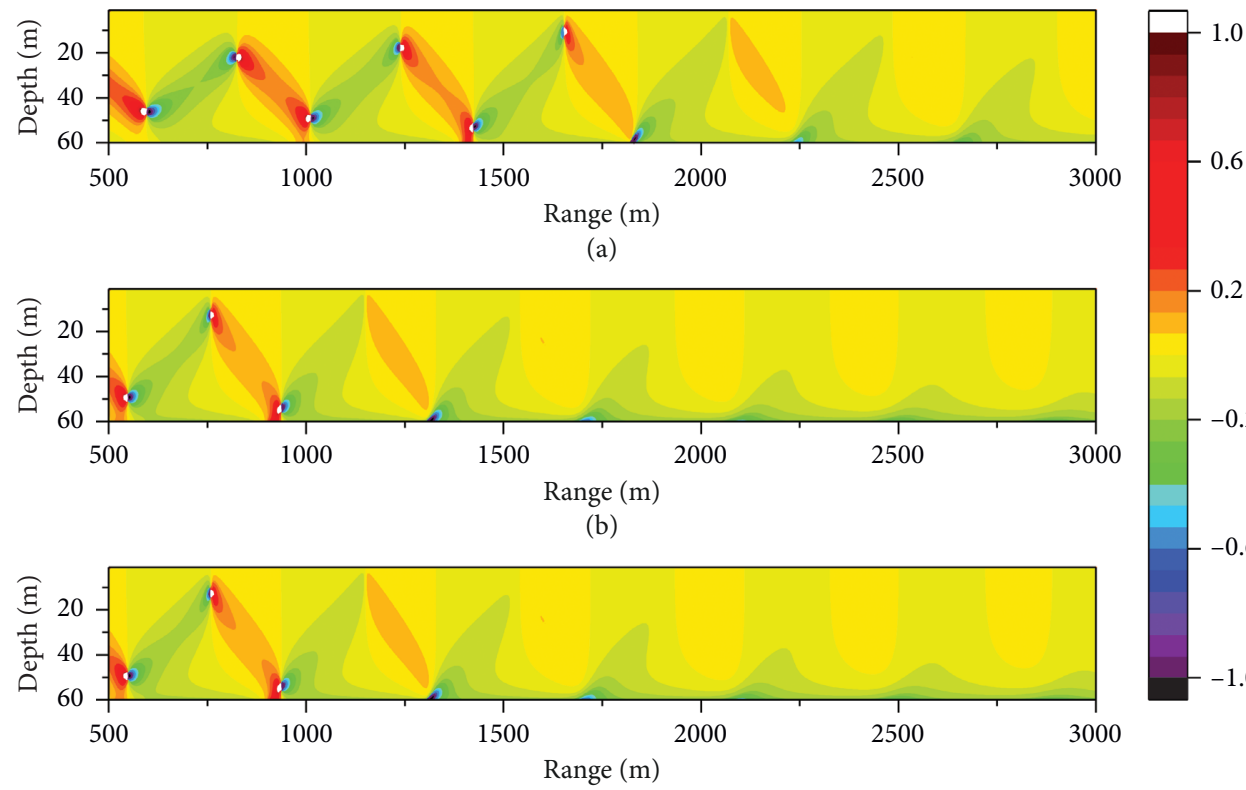

(c)

Figure 18: $I_{z}^{*}$ for different seabed densities when $f_{0}=50 \mathrm{~Hz}, h_{U}=20 \mathrm{~m}, c_{1}=1800 \mathrm{~m} / \mathrm{s}$, and $c_{s}=800 \mathrm{~m} / \mathrm{s}\left((\mathrm{a}) \rho_{1}=1600 \mathrm{~kg} / \mathrm{m}^{3} ;(\mathrm{b})\right.$ $\rho_{1}=2000 \mathrm{~kg} / \mathrm{m}^{3}$; (c) $\rho_{1}=3000 \mathrm{~kg} / \mathrm{m}^{3}$ ).

Finally, the influence of the seismic wave attenuations is analyzed. In this paper, $\alpha_{p}$ and $\alpha_{s}$ are defined as the compressional and shear wave attenuations in decibels per wavelength.
As shown in Figure 21, it is obvious that, with larger seismic wave attenuations, the normal modes attenuate more rapidly so that the interference effect is waken. 


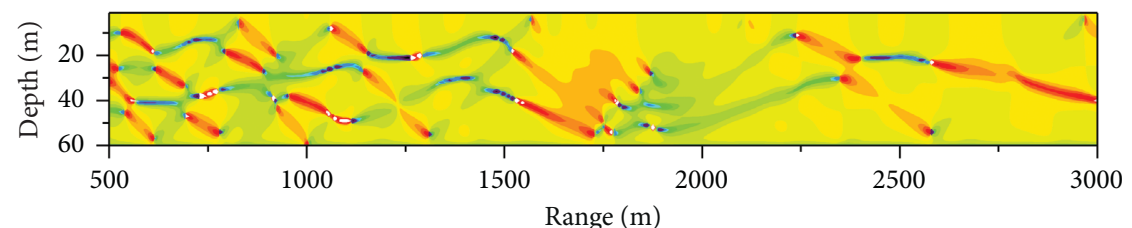

(a)

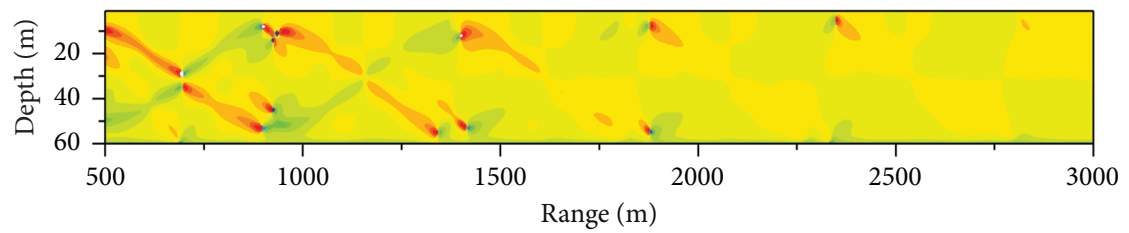

(b)

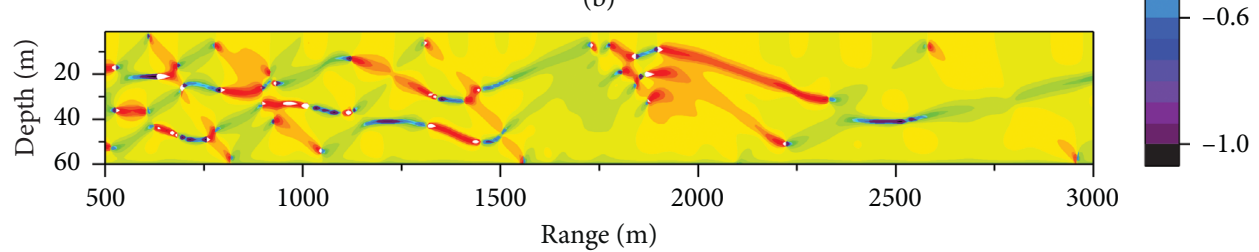

(c)

Figure 19: $I_{z}^{*}$ for different submerge depths when $f_{0}=190 \mathrm{~Hz}, \rho_{1}=1600 \mathrm{~kg} / \mathrm{m}^{3}, c_{1}=1800 \mathrm{~m} / \mathrm{s}$, and $c_{s}=800 \mathrm{~m} / \mathrm{s}\left((\mathrm{a}) h_{U}=5 \mathrm{~m}\right.$; $(\mathrm{b})$ $\left.h_{U}=30 \mathrm{~m} ;(\mathrm{c}) h_{U}=55 \mathrm{~m}\right)$.

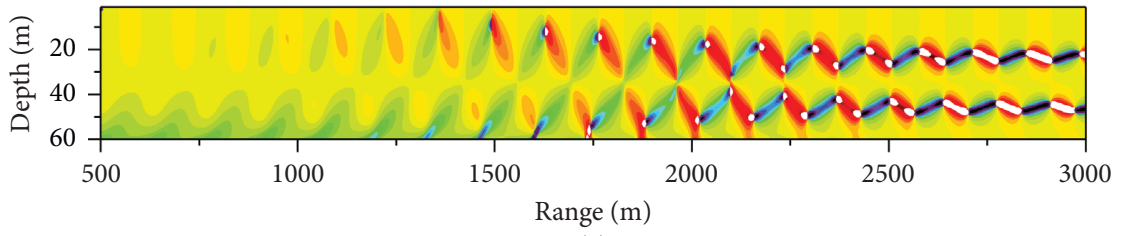

(a)

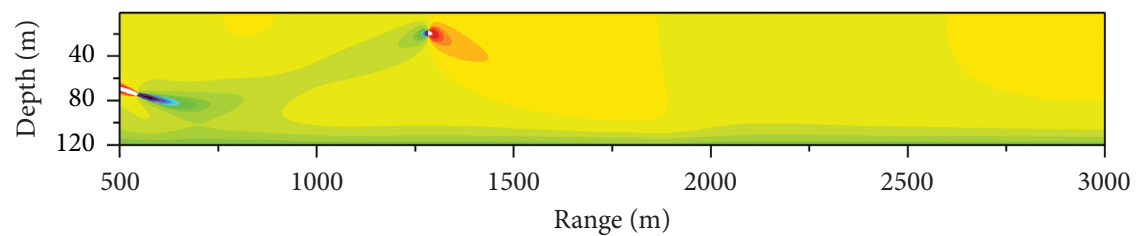

(b)

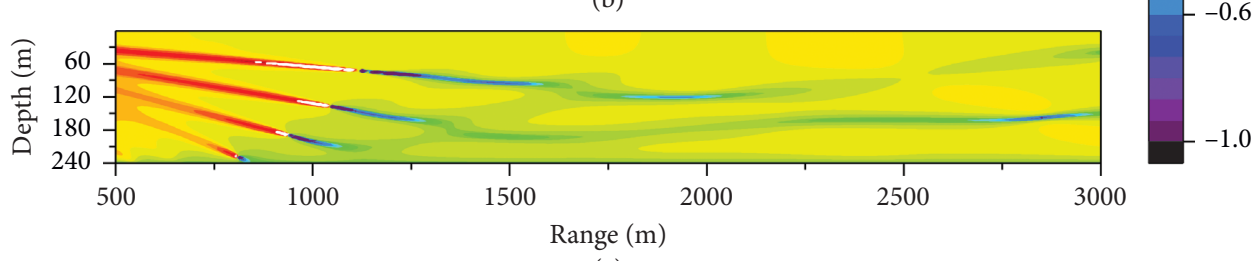

(c)

Figure 20: $I_{z}^{*}$ for different waveguide depths when $f_{0}=50 \mathrm{~Hz}, \rho_{1}=1600 \mathrm{~kg} / \mathrm{m}^{3}, c_{1}=2000 \mathrm{~m} / \mathrm{s}$, and $c_{s}=1200 \mathrm{~m} / \mathrm{s}((\mathrm{a}) H=60 \mathrm{~m}$; (b) $H=120 \mathrm{~m}$; (c) $H=240 \mathrm{~m}$ ). 


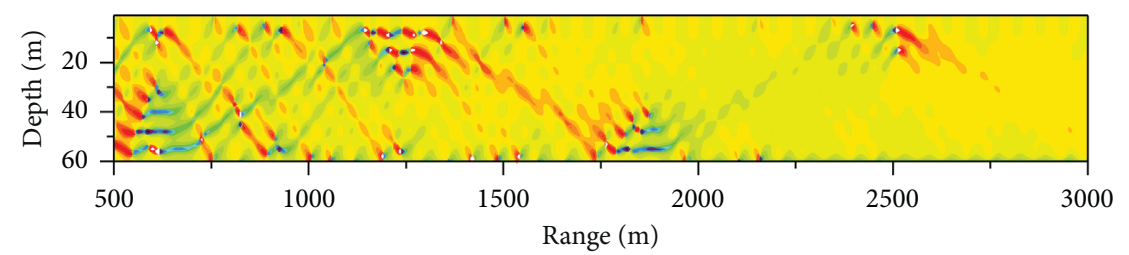

(a)

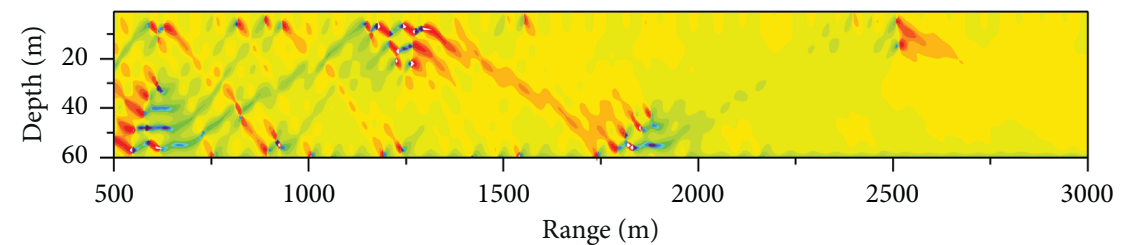

(b)

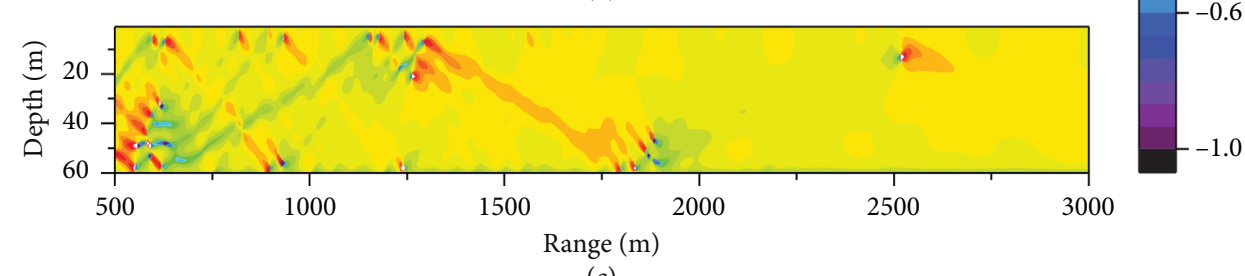

(c)

Figure 21: $I_{z}^{*}$ for different wave attenuations when $f_{0}=360 \mathrm{~Hz}, \rho_{1}=1600 \mathrm{~kg} / \mathrm{m}^{3}, c_{1}=1800 \mathrm{~m} / \mathrm{s}$, and $c_{s}=800 \mathrm{~m} / \mathrm{s}\left((\mathrm{a}) \alpha_{p}=\alpha_{s}=0.1 \mathrm{~dB} / \lambda\right.$; (b) $\alpha_{p}=\alpha_{s}=0.5 \mathrm{~dB} / \lambda$; (c) $\alpha_{p}=\alpha_{s}=1 \mathrm{~dB} / \lambda$ ).

Therefore, the quantity of vortices becomes smaller and the sound energy fluctuates less acutely at a long distance.

\section{Conclusion and Perspectives}

In this paper, a numerical method based on the CWSM is proposed for predicting the vector sound field of an elastic structure in a shallow water waveguide. The transfer function in the waveguide is modified to establish the relationship between equivalent source intensities and particle velocities in the field. Meanwhile, an approximate method is put forward for solving the equivalent source intensities. In addition, the acoustic intensity vector sound field characteristics of a cylindrical shell in a Pekeris waveguide are investigated. Some conclusions could be drawn as follows:

(1) By comparing the results of the CWSM and the FEM-BEM, the validity of the CWSM for predicting the vector sound field of a structure has been manifested. Compared with the FEM-BEM, the CWSM needs less information as the acoustical input, making it more feasible to implement in actual engineering. Meanwhile, the CWSM avoids the solution of singular integral which is inevitable in the FEM-BEM and reduces the computational cost dramatically.

(2) The result of equivalent source intensities could be convergent with finite orders of image sources considered. When the structure is quite close to the boundaries of the waveguide, the image sources have a significant effect on the equivalent source intensities. Nevertheless, only the first few orders of image sources are necessary to be considered at low frequencies. However, when the source is not close to the boundaries, the influence of the image sources on the equivalent source intensities could be neglected. With the above approximations, the computational cost is further reduced.

(3) At a long distance, the spatial structure of sound field is primarily determined by reflected sounds. Thus, vortices in the acoustic intensity field are mainly caused by the destructive interferences among different normal modes. The more normal modes exist in the waveguide, the more complicated the interferences are. Generally, though the similarity is demonstrated in the scalar sound field, the acoustic intensity vector field characteristics could be entirely different for a point source and an elastic structure. However, the high-order modes often attenuate rapidly with the increase of horizontal distance, especially when there are shear waves in the seabed. Usually, when the distance is long enough and there is only one mode in the sound field, the acoustic intensity characteristics are semblable for the two types of sources. Meanwhile, the interactions among the wavefronts coming from different equivalent sources could be the major causes of the vortices at the near field. Under the circumstances, the direct sounds have stronger effect on the spatial structure of sound field. Obvious discrepancies always exist for a point source and a structure at the near field.

(4) Source parameters, ocean environment parameters, and geo parameters could have effect on the acoustic intensity vector field. With the increase of source frequency, waveguide depth, and the seismic wave speeds, the mode quantity increases evidently which means that the interference effect is stronger and 
vortices are more likely to form in the acoustic intensity vector field. Meanwhile, though submerge depth does not affect the mode quantity, the spatial structures of acoustic intensity vector field could be different. When the source is close to the central depth of a waveguide, the sound energy distribution is more homogeneous and quite few vortices form under the circumstance. However, when the source is close to the boundaries of a waveguide, sound energy fluctuates more obviously and more vortices appear. The seabed density and seismic wave attenuations are not the determining factor for the spatial structure of sound field. Instead, they just influence the details of the sound field, especially at a long distance. In addition, it shows that, in a waveguide with an elastic seabed, the attenuation coefficient of a high-order mode which is close to "leaky modes" could be very small, even less than that of a low-order mode. Therefore, high-order modes could exist in the waveguide at quite a long distance to create vortices under specific circumstances.

The method presented in this paper provides a new approach to acquire the vector sound field characteristics of elastic structures in shallow water waveguides with high efficiency. Furthermore, it could have intensive usage in noise measurement, sound prediction, feature extraction, etc. In some extent, it may promote the development of vector hydrophone and technology of vector signal processing. However, still some complicated conditions such as coarse boundaries and nonparallel waveguide are not analyzed in this paper. Thus, we anticipate more comprehensive research about the issue to meet engineering requirement better.

\section{Appendix}

The reflection coefficient of an elastic seabed is defined as follows:

$$
V=\frac{\varsigma-1}{\varsigma+1} .
$$

The expression of $\varsigma$ is

$$
\varsigma=\rho_{1} \gamma_{\alpha 0} \frac{\left(\gamma_{1}-1\right)^{2}+\gamma_{1} \gamma_{B 1} \gamma_{\alpha 1}}{\rho_{0} \gamma_{\alpha 1}} .
$$

The variants in (A.2) are defined as below:

$$
\gamma_{\alpha 0}=\sqrt{\left(\frac{c}{c_{0}}\right)^{2}-1},
$$

where $c$ is defined as the horizontal wave speed in the water, $c=\omega_{0} / k$, and $k$ is defined as the horizontal wave number, $k=k_{0} \cos \theta_{n}$.

$$
\gamma_{\alpha 1}=\sqrt{\frac{\rho_{1} c^{2}}{\lambda_{1}+2 \mu_{1}}-1}
$$

where $\lambda_{1}$ and $\mu_{1}$ are the lame constants of the seabed and defined as follows:

$$
\begin{aligned}
\left(\lambda_{1}+2 \mu_{1}\right) & =\frac{\rho_{1}\left(x_{p}^{2}-y_{p}^{2}-j 2 x_{p} y_{p}\right)}{\left(x_{p}^{2}+y_{p}^{2}\right)^{2}}, \\
\mu & =\frac{\rho_{1}\left(x_{s}^{2}-y_{s}^{2}-j 2 x_{s} y_{s}\right)}{\left(x_{s}^{2}+y_{s}^{2}\right)^{2}},
\end{aligned}
$$

where $x_{p}=1 / c_{1}, \quad x_{s}=1 / c_{s}, \quad y_{p}=\alpha_{p} /\left(8.686 \omega_{0}\right), \quad y_{s}=\alpha_{s} /$ $\left(8.686 \omega_{0}\right)$,

$$
\begin{aligned}
\gamma_{B 1} & =\sqrt{\frac{\rho_{1} c^{2}}{\mu_{1}}-1}, \\
\gamma_{1} & =\frac{2 \mu_{1}}{\rho_{1} c^{2}} .
\end{aligned}
$$

Green's function in the waveguide with an elastic seabed could be written as follows:

$$
g(r, z)=\frac{p_{p}(r, z)}{4 \pi}
$$

where $p_{p}(r, z)$ is defined as the sound pressure of a point source.

$$
p_{p}(r, z)=\rho_{1} \omega_{0}^{2} \Phi_{p}(r, z),
$$

where $\Phi_{p}(r, z)$ is the potential function of a point source and defined as follows:

$$
\Phi_{p}(r, z)=\int_{0} Z_{1}(z, \xi) H_{0}^{(2)}(\xi r) \xi d \xi .
$$

The expression of $Z_{1}(z, \xi)$ could be written as follows:

$$
Z_{1}(z, \xi)=\left\{\begin{array}{l}
A^{\prime} \sin \left(k_{z} z\right), \quad 0<z<z_{0}, \\
B^{\prime} \sin \left(k_{z} z\right)+C^{\prime} \cos \left(k_{z} z\right), \quad z_{0}<z<H,
\end{array}\right.
$$

where $A^{\prime}$ and $B^{\prime}$ are undetermined coefficient:

$$
\begin{aligned}
& A^{\prime}=\frac{2}{k_{z}}\left[\frac{k_{z} \cos k_{z}\left(H-z_{0}\right)-j M \beta K \sin k_{z}\left(H-z_{0}\right)}{k_{z} \cos k_{z} H-j M \beta K \sin k_{z} H}\right], \\
& B^{\prime}=\frac{2 \sin k_{z} z_{0}}{k_{z}}\left[\frac{k_{z} \sin k_{z} H+j M \beta K \cos k_{z} H}{k_{z} \cos k_{z} H-j M \beta K \sin k_{z} H}\right],
\end{aligned}
$$

where $k_{1}=\omega_{0} / c_{1}$ and $\beta=\sqrt{k_{1}^{2}-\xi^{2}}$. The expression of $K$ is

$$
K=\frac{k_{s}^{4}}{4 \xi^{2}\left(\sigma^{2}+\beta \gamma\right)},
$$

where $k_{s}=\omega_{0} / c_{s}, \gamma=\sqrt{k_{s}^{2}-\xi^{2}}$, and $\sigma=\left(2 \xi^{2}-k_{s}^{2}\right) / 2 \xi$.

\section{Data Availability}

The data used to support the findings are available from the first author upon request. 


\section{Conflicts of Interest}

The authors declare that they have no conflicts of interest.

\section{Acknowledgments}

The authors acknowledge the financial support provided by the National Nature Science Foundation of China (Grant no. 11674074).

\section{References}

[1] Y. Zhang, The Effect and Application of Acoustic Photoelectric Waveguide, Publishing House of Electronics Industry, Beijing, China, 2014, in Chinese.

[2] T. W. Wu, "On computational aspects of the boundary element method for acoustic radiation and scattering in a perfect waveguide," The Journal of the Acoustical Society of America, vol. 96, no. 6, pp. 3733-3743, 1994.

[3] Z. Bai, W. Wu, C. Zuo et al., "Sound radiation and spread characteristics of cylindrical shell in finite depth water," Journal of Ship Mechanics, vol. 18, no. 1-2, pp. 178-190, 2014, in Chinese.

[4] T. Li, F. Jiang, W. Ye et al., "The wave characteristics of the acoustic radiation from cylindrical shells within finite depth from the free surface," Chinese Journal of Ship Research, vol. 8, no. 1, pp. 73-79, 2013, in Chinese.

[5] W. Guo, T. Li, X. Zhu, Y. Miao, and G. Zhang, "Vibration and acoustic radiation of a finite cylindrical shell submerged at finite depth from the free surface," Journal of Sound and Vibration, vol. 393, pp. 338-352, 2017.

[6] W. Guo, T. Li, X. Zhu et al., "Analytical research of vibration and far-field acoustic radiation of cylindrical shell immersed at finite depth," Chinese Journal of Ship Research, vol. 12, no. 4, pp. 62-70, 2017, in Chinese.

[7] Y. Miao, T. Li, X. Zhu et al., "Research on the acoustical radiation characteristics of cylindrical shells in a shallow sea," Journal of Harbin Engineering University, vol. 38, no. 5, pp. 719-726, 2017, in Chinese.

[8] P. Wang, T.-Y. Li, X. Zhu, W.-J. Guo, and R. Nie, “An analytical solution for free flexural vibration of a thin cylindrical shell submerged in acoustic half-space bounded by a free surface," International Journal of Structural Stability and Dynamics, vol. 18, no. 3, p. 18, Article ID 1850042, 2018.

[9] G. H. Koopmann and J. Fahnline, "A method for computing acoustic fields based on the principle of wave superposition," The Journal of the Acoustical Society of America, vol. 86, no. 6, pp. 2433-2438, 1989.

[10] R. Jeans and I. C. Mathews, "The wave superposition method as a robust technique for computing acoustic fields," The Journal of the Acoustical Society of America, vol. 92, no. 2, pp. 1156-1166, 1992.

[11] J. Y. Hwang and S. C. Chang, "A retracted boundary integral equation for exterior acoustic problem with unique solution for all wave numbers," The Journal of the Acoustical Society of America, vol. 90, no. 2, pp. 1167-1180, 1991.

[12] J. Fahnline and G. Koopmann, "A numerical solution for the general radiation problem based on the combined methods of superposition and singular-value decomposition," The Journal of Acoustic Society of America, vol. 90, no. 2, pp. 28082819, 1991.

[13] J. Li, J. Chen, C. Yang et al., "Analysis of the impact factors on the accuracy of sound field reconstruction based on wave superposition," Acta Physica Sinica, vol. 57, no. 7, pp. 42584264, 2008, in Chinese.

[14] H. Chen, D. Shang, Q. Li et al., "Sound radiation prediction for underwater structure by field-matching wave superposition method," Acta Acustica, vol. 38, no. 2, pp. 137-146, 2013, in Chinese.

[15] H. Chen and D. Shang, "Fast prediction of acoustic radiation from a hemi-capped cylindrical shell in waveguide," Journal of Marine Science and Application, vol. 4, no. 13, pp. 437-448, 2014.

[16] Y. Wang, Theoretical and Experimental Research of Underwater Noise Reduction Prediction Based on Wave Superposition Method, Harbin Engineering University, Harbin, China, 2013, in Chinese.

[17] H. Chen, Research on Prediction of Sound Radiated by Elastic Structure in Underwater Bounded Space, Harbin Engineering University, Harbin, China, 2013, in Chinese.

[18] D. Shang, Z. Qian, Y. He et al., "Sound radiation of cylinder in shallow water investigated by combined wave superposition method," Acta Physica Sinica, vol. 67, no. 8, Article ID 084301, pp. 1-13, 2018, in Chinese.

[19] Z. Qian, Research on Acoustic Radiation Prediction Method for Cylindrical Shell in the Shallow Water, Harbin Engineering University, Harbin, China, 2018, in Chinese.

[20] S. Zhou, "Acoustic vector fields propagation in horizontal stratified waveguide," Journal of Harbin Engineering University, vol. 25, no. 1, pp. 38-42, 2004, in Chinese.

[21] H. Zhu, G. Zheng, H. Zhang et al., "Study on propagation characteristics of low frequency acoustic signal in shallow water environment," Journal of Shanghai Jiao Tong University, vol. 51, no. 12, pp. 1464-1472, 2017, in Chinese.

[22] K. Qu, S. Piao, J. Zhou, and F. Zhu, "Analysis of surface sound duct in the northern shelf of the south China sea," Shock and Vibration, vol. 2018, Article ID 2409761, 12 pages, 2018.

[23] H. Ji, X. Xu, X. Guo, S. Ye, J. Chen, and X. Yang, "Direct FVM simulation for sound propagation in an ideal wedge," Shock and Vibration, vol. 2016, Article ID 3703974, 9 pages, 2016.

[24] H. Zhang, Research on Modeling and Rule of Infrasound Propagation in Shallow Sea, Harbin Engineering University, Harbin, China, 2010, in Chinese.

[25] D. Wang, Theoretical Study on Vector Acoustic Field and Vector Signal Processing, Harbin Engineering University, Harbin, China, 2004, in Chinese.

[26] M. Bayat, H. Ahmadi, I. Pakar et al., "Nonlinear frequency analysis of beams resting on elastic foundation using max-min approach," Geomechanics and Engineering, vol. 16, no. 4, pp. 355-361, 2018.

[27] G. Sun, Shallow Water Acoustic Vector Field and its Signal Processing, Harbin Engineering University, Harbin, China, 2008, in Chinese.

[28] G. M. Revel and G. L. Rossi, "Sound power estimation by laser Doppler vibration measurement techniques," Shock and Vibration, vol. 5, pp. 297-305, Article ID 232715, 1998.

[29] Y. Yun, J. Hui, A. Zhao et al., "Complex acoustic intensity of normal modes in Pekeris waveguide and its application," Acta Physica Sinica, vol. 57, no. 9, pp. 5742-5748, 2008, in Chinese.

[30] J. Hui, G. Sun, and A. Zhao, "Normal mode acoustic intensity flux in Pekeris waveguide and its cross spectra signal processing," Acta Acustica, vol. 33, no. 4, pp. 300-304, 2008, in Chinese.

[31] P. Hu, The Study on Measurement of Radiated Noise from Submarine Due to Inhomogeneous Distribution of Acoustic Energy in Shallow Sea, Harbin Engineering University, Harbin, China, 2010, in Chinese. 
[32] O. Dall, R. David, P. Dahl et al., "Properties of the acoustic intensity vector field in a shallow water waveguide," The Journal of Acoustic Society of America, vol. 131, no. 3, pp. 2023-2035, 2012.

[33] H. Zhu, S. Piao, H. Zhang et al., "An extraction method for the interference striation of acoustic vector fields in shallow water," Acta Acustica, vol. 41, no. 1, pp. 30-40, 2016, in Chinese.

[34] F. Jacobsen, "Sound field indicators: useful tools," Noise Control Engineering Journal, vol. 35, no. 1, pp. 37-46, 1990. 